\title{
Phase Retrieval from Modulus Using Homeomorphic Signal Processing and the Complex Cepstrum: An Algorithm for Lightning Protection Systems
}

Grace A. Clark

June 8, 2004

U.S. Department of Energy

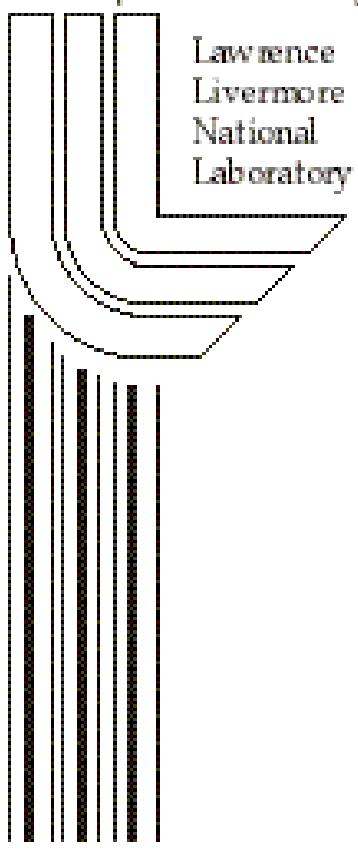




\section{DISCLAIMER}

This document was prepared as an account of work sponsored by an agency of the United States Government. Neither the United States Government nor the University of California nor any of their employees, makes any warranty, express or implied, or assumes any legal liability or responsibility for the accuracy, completeness, or usefulness of any information, apparatus, product, or process disclosed, or represents that its use would not infringe privately owned rights. Reference herein to any specific commercial product, process, or service by trade name, trademark, manufacturer, or otherwise, does not necessarily constitute or imply its endorsement, recommendation, or favoring by the United States Government or the University of California. The views and opinions of authors expressed herein do not necessarily state or reflect those of the United States Government or the University of California, and shall not be used for advertising or product endorsement purposes.

This work was performed under the auspices of the U. S. Department of Energy by the University of California, Lawrence Livermore National Laboratory under Contract No. W-7405-Eng-48.

This report has been reproduced directly from the best available copy.

Available electronically at http://www.doc.gov/bridge

Available for a processing fee to U.S. Department of Energy

And its contractors in paper from

U.S. Department of Energy

Office of Scientific and Technical Information

P.O. Box 62

Oak Ridge, TN 37831-0062

Telephone: (865) 576-8401

Facsimile: (865) 576-5728

E-mail: reports@adonis.osti.gov

Available for the sale to the public from

U.S. Department of Commerce

National Technical Information Service

5285 Port Royal Road

Springfield, VA 22161

Telephone: (800) 553-6847

Facsimile: (703) 605-6900

E-mail: orders@ntis.fedworld.gov

Online ordering: http://www.ntis.gov/ordering.htm

OR

Lawrence Livermore National Laboratory

Technical Information Department's Digital Library

http: / / www.llnl.gov/tid/Library.html 


\title{
Phase Retrieval from Modulus Using Homeomorphic Signal Processing and the Complex Cepstrum: \\ An Algorithm for Lightning Protection Systems
}

\author{
Grace A. Clark, Ph.D. \\ Lawrence Livermore National Laboratory \\ 7000 East Ave., L-130, Livermore, CA 94550 \\ (925) 423-9759 (Office), (925) 422-2495 (FAX), \\ clark9@1lnl.gov
}

June 8, 2004

UCRL-TR-205254 


\section{Acknowledgments}

The author gratefully acknowledges the important contributions to this work made by her colleagues. Dr. Mike M. Ong and Dr. Charles G. Brown of the Defense Sciences Engineering Division (DSED) motivated the work as part of their Lightning Protection projects. In particular, Charles discussed with me the specific currently-used signal processing algorithms and their applications. Mike, on behalf of the Lightning Protection Project for Pantex, funded this work for about two weeks of my time. 


\begin{abstract}
In general, the Phase Retrieval from Modulus problem is very difficult [16]. In this report, we solve the difficult, but somewhat more tractable case in which we constrain the solution to a minimum phase reconstruction. We exploit the real-and imaginary part sufficiency properties of the Fourier and Hilbert Transforms of causal sequences to develop an algorithm for reconstructing spectral phase given only spectral modulus. The algorithm uses homeomorphic signal processing methods with the complex cepstrum. The formal problem of interest is: Given measurements of only the modulus $|H(k)|$ (no phase) of the Discrete Fourier Transform (DFT) of a real, finite-length, stable, causal time domain signal $h(n)$, compute a minimum phase reconstruction $\widehat{h}(n)$ of the signal. Then compute the phase of $\widehat{h}(n)$ using a DFT, and exploit the result as an estimate of the phase of $h(n)$. The development of the algorithm is quite involved, but the final algorithm and its implementation are very simple.

This work was motivated by a Phase Retrieval from Modulus Problem that arose in LLNL Defense Sciences Engineering Division (DSED) projects in lightning protection for buildings. The measurements are limited to modulus-only spectra from a spectrum analyzer. However, it is desired to perform system identification on the building to compute impulse responses and transfer functions that describe the amount of lightning energy that will be transferred from the outside of the building to the inside. This calculation requires knowledge of the entire signals (both modulus and phase). The algorithm and software described in this report are proposed as an approach to phase retrieval that can be used for programmatic needs.
\end{abstract}

This report presents a brief tutorial description of the mathematical problem and the derivation of the phase retrieval algorithm. The efficacy of the theory is demonstrated using simulated signals that meet the assumptions of the algorithm. We see that for the noiseless case, the reconstructions are extremely accurate. When moderate to heavy simulated white Gaussian noise was added, the algorithm performance remained reasonably robust, especially in the low frequency part of the spectrum, which is the part of most interest for lightning protection.

Limitations of the algorithm include the following: (1) It does not account for noise in the given spectral modulus. Fortunately, the lightning protection signals of interest generally have a reasonably high signal-to-noise ratio (SNR). (2) The DFT length $N$ must be even and larger than the length of the nonzero part of the measured signals. These constraints are simple to meet in practice. (3) Regardless of the properties of the actual signal $h(n)$, the phase retrieval results are constrained to have the minimum phase property. In most problems of practical interest, these assumptions are very reasonable and probably valid. They are reasonable assumptions for Lightning Protection applications.

Proposed future work includes (a) Evaluating the efficacy of the algorithm with real Lightning Protection signals from programmatic applications, (b) Performing a more rigorous analysis of noise effects, (c) Using the algorithm along with advanced system identification algorithms to estimate impulse responses and transfer functions, (d) Developing algorithms to deal with measured partial (truncated) spectral moduli, and (e) R \& D of phase retrieval algorithms that specifically deal with general (not necessarily minimum phase) signals, and noisy spectral moduli. 


\section{Contents}

1 Introduction $\quad 5$

2 Theoretical Background $\quad 7$

2.1 Homeomorphism and the Complex Cepstrum $\ldots \ldots \ldots \ldots$

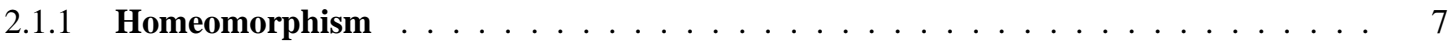

2.1.2 Homeomorphic Signal Processing $\ldots \ldots \ldots \ldots \ldots \ldots$

2.1.3 The Complex Cepstrum and the Real Cepstrum $\ldots \ldots \ldots \ldots \ldots$

2.1 .4 A Note on Terminology $\ldots \ldots \ldots \ldots \ldots \ldots$

2.1.5 Properties of the Complex Cepstrum $\ldots \ldots \ldots \ldots$

2.2 The Hilbert Transform $\ldots \ldots \ldots \ldots$

2.3 Properties of Fourier and Hilbert Transforms $\ldots \ldots \ldots \ldots \ldots$

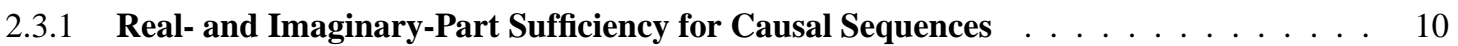

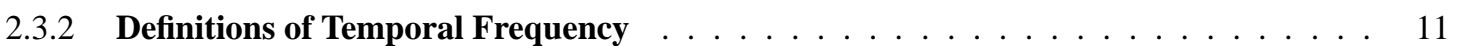

2.3.3 Relationship Between the Fourier and Hilbert Transforms . . . . . . . . . . . . . 11

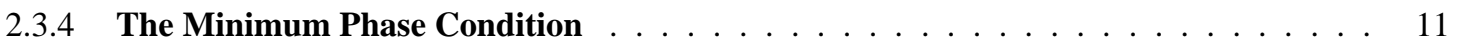

2.4 Discrete Fourier Transform Properties and the Hilbert Transform ～. . . . . . . . . . . 12

2.5 Realization of the Complex Cepstrum Using the Complex Logarithm ～. . . . . . . . . . 14

2.6 Minimum Phase Realization Given a Finite-Length Sequence . . . . . . . . . . . . . . . . 14

3 Phase Reconstruction Given Only the Spectral Modulus $\quad 18$

3.0.1 Construct an Estimate of the Minimum Phase Realization Given Only the Spectral Modulus . . . . . . . . . . . . . . . . . . . . . . . . . . 18

3.0.2 Reconstruct the Phase from the Estimated Complex Cepstrum _ . . . . . . . . . . 19

3.1 Relationship with the Minimum Phase Realization Algorithm . . . . . . . . . . . . . . . . . . 19

3.2 Limitations of the Algorithm $\ldots \ldots \ldots \ldots$

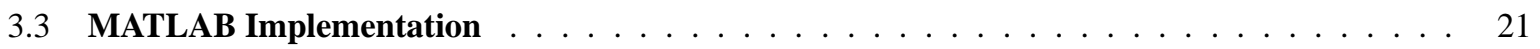


4 Simulation Experiments 22

4.1 Experimental Approach . . . . . . . . . . . . . . . . . . . . . . . . . 22

4.2 Definitions for the Simulations . . . . . . . . . . . . . . . . . . . . . . 22

4.2.1 Define the Noiseless Simulated Signal . . . . . . . . . . . . . . . . . . . . . . . . 22

4.2 .2 The Simulated Lightning Signal . . . . . . . . . . . . . . . . . . . . . . . . . 23

4.2.3 Define the Simulated Noisy Signals _ . . . . . . . . . . . . . . . . . . . . . . . 23

4.2.4 Define Noise and Signal-to-Noise Ratio (SNR) . . . . . . . . . . . . . . . . . . . . . 24

4.3 Simulation Example for the Noiseless Case . . . . . . . . . . . . . . . . . . . . . . . 25

4.4 Simulation Example For SNR = 1.e-5 (50 dB) . . . . . . . . . . . . . . . . . . . . . . . 28

4.5 Simulation Example For $\mathrm{SNR}=1 . \mathrm{e} 4(\mathbf{4 0} \mathrm{dB}) \ldots \ldots \ldots \ldots$

5 Future Work $\quad 34$

5.1 Evaluation of the Algorithm Using Measured Lightning Protection Moduli . . . . . . . . . . 34

5.2 Apply Optimal Least-Squares Impulse Response Estimation Algorithms for System Identification . . . . . . . . . . . . . . . . . . . . . . . . . . . . . . . . 34

5.3 Phase Retrieval for Partial Moduli . . . . . . . . . . . . . . . . . . . . . . . . . . . . . . 34

$5.4 \quad$ R\&D of Generalized Phase Retrieval Algorithms . . . . . . . . . . . . . . . . . . . . . . . 35

6 Conclusions $\quad 36$

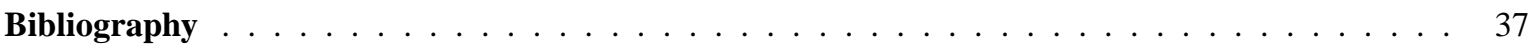




\section{List of Figures}

2.1 Block Diagram of the Minimum Phase Reconstruction Algorithm. Given a finite-length, real causal, stable sequence $h(n)$, we can construct a minimum phase realization $\widehat{h}(n)$ of $h(n)$. The signal $\widehat{h}(n)$ is an estimate of the complex cepstrum of $h(n) \ldots \ldots \ldots \ldots \ldots$

3.1 Block Diagram of the proposed Phase Reconstruction Algorithm. Given the input spectral modulus $|H(k)|$, we can construct a finite-length, real, causal, stable sequence $\widehat{h}(n)$ corresponding to that spectral modulus. The signal $\widehat{h}(n)$ is a minimum phase reconstruction of $h(n)$, and it is an estimate of the complex cepstrum of $h(n) \ldots \ldots \ldots \ldots \ldots \ldots$

4.1 (Upper Left): The simulated noiseless minimum phase signal $x(n), N=128$. Clearly, this finitelength signal is real, causal and stable. (Upper Right): The magnitude squared of the N-point DFT of the noiseless signal $x(n)$. (Lower Left): The magnitude and phase of the N-point DFT of the noiseless signal $\mathrm{x}(\mathrm{n})$. (Lower Right): $\mathrm{c}(\mathrm{n})=$ The real cepstrum $\widehat{h}(n)$ of $\mathrm{h}(\mathrm{n})$ for the noiseless case.

4.2 (Upper Left): The reconstructed minimum phase signal $\widehat{h}(n)$ for the noiseless case $(N=128)$. (Upper Right): The signal $\mathrm{x}(\mathrm{n})$ overlayed with the reconstructed signal $\widehat{h}(n)$ for the noiseless case. The agreement between the two is excellent $(N=128)$. (Lower Left): Magnitude squared of the DFT of the reconstructed signal $\widehat{h}(n)$ for the noiseless case $(N=128)$. (Lower Right): Magnitude $|\widehat{H}(k)|$ and phase $\arg [\widehat{H}(k)]$ for the noiseless case $(N=128) \ldots \ldots \ldots \ldots$

4.3 Magnitude $|X(k)|$ and phase $\arg [X(k)]$ overlayed on the reconstructed magnitude $|\widehat{H}(k)|$ and phase $\arg [\widehat{H}(k)]$ for the noiseless case $(N=128)$. The agreement is excellent. . . . . . . . .

4.4 (Upper Left): The noisy minimum phase signal $\mathrm{z}(\mathrm{n})$ for $\mathrm{SNR}=1 . \mathrm{e} 5(50 \mathrm{~dB})$. (Upper Right): The magnitude squared of the DFT of the noisy signal $z(n)$ for $S N R=50 \mathrm{~dB}$. (Lower Left): The magnitude and phase of the DFT of the noisy signal $\mathrm{z}(\mathrm{n})$ for $\mathrm{SNR}=50 \mathrm{~dB}$. (Lower Right): $\mathrm{c}(\mathrm{n})=$ the Real Cepstrum of $\mathrm{h}(\mathrm{n})$ for $\mathrm{SNR}=50 \mathrm{~dB}$

4.5 (Upper Left): The Reconstructed Minimum Phase Signal $\widehat{h}(n)$ when $\mathrm{SNR}(\mathrm{dB})=50 \mathrm{~dB}$. (Upper Right): The noiseless signal $\mathrm{x}(\mathrm{n})$ and the Reconstructed Signal $\widehat{h}(n)$ for SNR $=50 \mathrm{~dB}$. (Lower Left): The magnitude squared of the DFT of the Reconstructed Signal $\widehat{h}(n)$ for SNR $=50 \mathrm{~dB}$. (Lower Right): The magnitude and phase of the DFT of the reconstructed signal $\widehat{h}(n)$

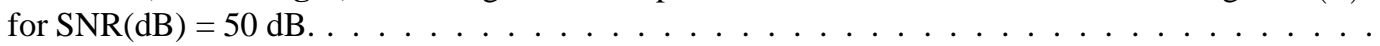

4.6 The noiseless magnitude and phase of $\mathrm{X}(\mathrm{k})$ and the reconstructed magnitude and phase of $\widehat{H}(k)$

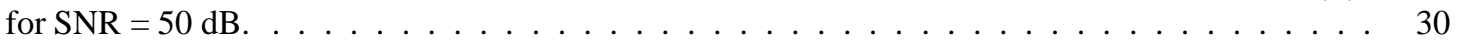

4.7 The noisy minimum phase signal $\mathrm{z}(\mathrm{n})$ for $\mathrm{SNR}=1 . \mathrm{e} 4(40 \mathrm{~dB}) \ldots \ldots \ldots \ldots \ldots \ldots$ 
4.8 The noiseless magnitude and phase of $\mathrm{X}(\mathrm{k})$ and the reconstructed magnitude and phase of $\widehat{H}(k)$ for $\mathrm{SNR}(\mathrm{dB})=40 \mathrm{~dB}$. The errors in the phase estimate appear to be small at low frequencies. They are much larger closer to the folding frequency. . . . . . . . . . . . . . . . . 


\section{Chapter 1}

\section{Introduction}

This report describes a homeomorphic algorithm for phase retrieval from modulus and MATLAB software for implementing it. The algorithm exploits important properties of the Fourier Transform, the Hilbert Transform and the Complex Cepstrum. The formal problem of interest is: Given measurements of only the modulus $|H(k)|$ (no phase) of the Discrete Fourier Transform (DFT) of a real, finite-length, stable, causal time domain signal $h(n)$, compute a minimum phase reconstruction $\widehat{h}(n)$ of the signal. The phase of $\widehat{h}(n)$ then serves as an estimate of the phase of $h(n)$. In general, the problem of phase retrieval from modulus is very difficult [16]. In this report, we solve the difficult, but somewhat more tractable case in which we constrain the solution to a minimum phase reconstruction.

This work was motivated by a phase retrieval from modulus problem that arose in DSED projects in lightning protection for buildings [20,21, 22, 23]. The measurements are limited to magnitude-only spectra from a spectrum analyzer. However, it is desired to perform system identification on the building to compute impulse responses and transfer functions that describe the amount of lightning energy will be transferred from the outside of the building to the inside. This algorithm and software are proposed as an approach to phase retrieval that can be adapted for programmatic needs.

In the report [16], the author proposed three main approaches to solving the problem of estimating a signal given only its DFT modulus. (1) The first approach (short-term), was to apply the Parks-McClellan Remez Exchange algorithm for finite impulse response (FIR) filter design. This approach was applied by C. G. Brown in [22]. (2) The second approach (medium-term) was to explore the use of homeomorphic signal processing techniques using the complex cepstrum. That is the approach followed in this report. It is not designed to deal with noisy signals. (3) The third approach (long-term) was to embark upon a research and development program to create an advanced phase retrieval algorithm that is designed to deal with general (not necessarily minimum phase) signals, including noisy signals. The third approach is proposed for future work.

This report presents a brief tutorial description of the mathematical problem and the derivation of the phase retrieval algorithm. The derivation is quite involved, but the final algorithm and its implementation are very simple. The theoretical principles are demonstrated by results of processing simulated signals. A brief demonstration of the effects of additive white Gaussian noise (WGN) is given to show the level of robustness of the technique. Future recommended work includes applying this algorithm to real measured signals for the Lightning Protection program, and performing a thorough evaluation of performance under noisy conditions.

The main contribution of this report lies in two main areas: (1) Bringing together a great deal of theory that is distributed among a variety of references and documenting it in a tutorial fashion that facilitates the understanding of homeomorphic systems and the phase retrieval algorithm. This includes descriptions and algorithm block diagrams that are not found elsewhere, and (2) Applying the techniques in the distributed literature to create an algorithm useful for phase retrieval from modulus for lightning protection systems. 
This report is organized as follows. In Chapter 2, we present the theoretical background necessary to position ourselves for writing the proposed phase retrieval algorithm. This includes a statement of various Fourier Transform and Hilbert Transform properties having to do with real-and imaginary part sufficiency, Discrete Fourier Transform (DFT) properties, realization of the complex cepstrum using the complex logarithm and deriving a minimum phase realization using the DFT. In Chapter 3, we propose an algorithm for phase retrieval from modulus, based upon the ideas presented in Chapter 2. Chapter 4 describes simulation experiments designed to demonstrate the theoretical principles of the proposed algorithm. Chapters 5 and 6 present ideas for future work and conclusions, respectively. 


\section{Chapter 2}

\section{Theoretical Background}

In this section, we very briefly describe the theoretical basics that lead to a phase retrieval algorithm based upon the Discrete Fourier Transform (DFT). This theory is distributed about the literature, but summarized here in tutorial form. For more detail and the formal derivations of the background material, the reader is referred to the bibliography.

\subsection{Homeomorphism and the Complex Cepstrum}

\subsubsection{Homeomorphism}

Let us review the formal definitions of metric space and homeomorphism [25]:

If $X$ is a normed linear space and $x, y \in X$, it is easily verified that the function $d(x, y)=\|x-y\|$ satisfies the following conditions:

(1) $d(x, y) \geq 0$ and is equal to zero if and only if $x=y$.

(2) $d(x, y)=d(y, x)$;

(3) $d(x, z) \leq d(x, y)+d(y, z)$ for any $x, y, z \in X$ (triangle inequality).

DEFINITION: $X$, an arbitrary set will be called a metric space if there is a function $d: X \times X \rightarrow R$ satisfying properties (1)-(3) above. The mapping $d$ itself will be called a metric.

DEFINITION: Let $(X, d)$ and $\left(Y, d^{\prime}\right)$ be metric spaces and let $f$ be a one-to-one, onto mapping such that $f: X \rightarrow$ $Y$. If $f$ and $f^{-1}$ are each continuous functions (mappings of this type are called bicontinuous), then $f$ is said to be a homeomorphism. Further, $X$ is said to be homeomorphic to $Y$ if such a mapping exists.

Homeomorphisms preserve all the essential topological properties; in particular, open sets in $X$ are mapped into open sets in $Y$, and, if $x$ is a limit point of $A \subset X$, then $f(x)$ will be a limit point of $f(A)$.

\subsubsection{Homeomorphic Signal Processing}

Homeomorphic (or homomorphic) signal processing methods have been developed as a way to deal with classes of nonlinear systems that obey a generalized superposition principle. Such systems are represented by algebraically linear transformations between input and output vector spaces, and have thus been called homomorphic systems [1, $2,25]$. Two classes of homomorphic systems that are especially well-suited to signal processing are those in 
which signals are combined by multiplication and convolution. It can be shown that the problem of designing homomorphic systems for multiplication and convolution reduces to a problem of designing a linear system [1].

Homomorphic processing has been successfully applied to problems involving the superposition of multiple signals in a variety of applications, including seismic signal processing and speech processing $[1,2,10,11,12,13]$. In particular, the techniques typcially involve converting nonlinear systems with multiplicative effects into linear systems with additive effects through the logarithm.

\subsubsection{The Complex Cepstrum and the Real Cepstrum}

Here we give a preview of two defintitions we develop and use later in the paper. Given a discrete-time sequence $h(n)$ that has a corresponding z-transform $H(z)$, we can define the quantity $H(z)$ as follows:

$$
\widehat{H}(z)=\log [H(z)]
$$

As we shall see later, we define the complex cepstrum $\widehat{h}(n)$ as follows.

$$
\begin{aligned}
\widehat{h}(n) & =Z^{-1}[\widehat{H}(z)] \\
& =Z^{-1}\{\log [H(z)]\}
\end{aligned}
$$

In a later section, we show that the complex cepstrum $\widehat{h}(n)$ of a real sequence $h(n)$ can be evaluated by means of a Discrete Fourier Transform (DFT) as follows:

$$
\widehat{h}(n)=I D F T[\log \{D F T[h(n]\}]
$$

where the DFT length $N$ must be chosen to be sufficient to avoid cepstral aliasing. In addition, a complex logarithm must be used, and the phase must be unwrapped carefully [7].

Let us now consider taking the real logarithm of the modulus of the z-transform $H(z)$. Let

$$
\widehat{H}(z)=\log |H(z)|
$$

The real cepstrum $c(n)$ of the real sequence $h(n)$ is then

$$
\begin{aligned}
c(n) & =Z^{-1}\{\widehat{H}(z)\} \\
& =Z^{-1}\{\log |H(z)|\}
\end{aligned}
$$

In a later section, we show that the real cepstrum $c(n)$ of a real sequence $h(n)$ can be evaluated by means of a Discrete Fourier Transform (DFT) as follows:

$$
c(n)=I D F T[\log \mid D F T[h(n] \mid]
$$

where the DFT length $N$ must be chosen to be sufficient to avoid cepstral aliasing [7].

\subsubsection{A Note on Terminology}

The origin of the term cepstrum is discussed in [1]. The name "cepstrum "was coined by Bogert, Healy and Tukey in their 1963 paper [10]. In this paper, the authors were processing signals containing echoes. They found that the logarithm of the power spectrum of a signal containing an echo has an additive periodic component due to the echo. Therefore, the Fourier transform of the log-power spectrum should exhibit a peak at the echo delay. They 
called the log-power spectrum the cepstrum, as a variation of the word spectrum. They did this because, in their words, "In general, we find ourselves operating on the frequency side in ways customary on the time side and vice versa [10]." The authors continued defining a variety of new words, including alanysis corresponding to analysis, quefrency corresponding to frequency, lifter corresponding to filter, etc. Since then, the concepts have been used widely, but only the word cepstrum has been adopted widely.

In general, for cepstrum analysis, we must use the complex logarithm, the complex Fourier Transform, and the complex Discrete Fourier Transform. Therefore, to emphasize this point, the term complex cepstrum is used. It is also important to emphasize, however, that the complex cepstrum $\widehat{h}(n)$ is real when the inputs sequence $h(n)$ is real. In the literature, the term cepstrum is reserved only for use in the case in which the real logarithm is used [1].

\subsubsection{Properties of the Complex Cepstrum}

The following properties P1 through P4 of the complex cepstrum are derived in [1], p. 502.

(P1): The complex cepstrum decays at least as fast as $1 / n$ : Specifically,

$$
|\widehat{h}(n)|<C\left|\frac{\alpha^{n}}{n}\right|, \quad-\infty<n<\infty
$$

where $C$ is a constant and $\alpha$ equals the maximum of $\left|a_{k}\right|,\left|b_{k}\right|,\left|c_{k}\right|$, and $\left|d_{k}\right|$, the constants in a rational polynomial model of the z-transform $H(z)$ [1].

(P2): If $h(n)$ is minimum phase (no poles or zeros outside the unit circle), then

$$
\widehat{h}(n)=0, \quad n<0
$$

(P3): If $h(n)$ is maximum phase (no poles or zeros inside the unit circle), then

$$
\widehat{h}(n)=0, \quad n>0
$$

(P4): If $h(n)$ is of finite duration, $\widehat{h}(n)$ will nevertheless have infinite duration.

We shall see that these properties play a very important role in the derivation of a phase reconstruction algorithm.

\subsection{The Hilbert Transform}

Let $t$ denote the continuous time variable. Given a real-valued function $x(t)$ in the interval $-\infty<t<\infty$, its Hilbert Transform, denoted by $\widehat{x}(t)$ and its inverse are defined by $[3,2]$ :

$$
\begin{aligned}
& \widehat{x}(t)=\frac{1}{\pi} P \int_{-\infty}^{\infty} \frac{x(\tau)}{t-\tau} d \tau \quad=-\frac{1}{\pi} P \int_{-\infty}^{\infty} \frac{x(t+\tau)}{\tau} d \tau=\frac{1}{\pi} P \int_{-\infty}^{\infty} \frac{x(t-\tau)}{\tau} d \tau \\
& x(t)=-\frac{1}{\pi} P \int_{-\infty}^{\infty} \frac{\widehat{x}(\tau)}{t-\tau} d \tau \quad=\frac{1}{\pi} P \int_{-\infty}^{\infty} \frac{\widehat{x}(t+\tau)}{\tau} d \tau=-\frac{1}{\pi} P \int_{-\infty}^{\infty} \frac{\widehat{x}(t-\tau)}{\tau} d \tau
\end{aligned}
$$

where the symbol P denotes the Cauchy Principle Value of the integral [25, 3]. By the Cauchy Principle Value of an integral $P \int_{-\infty}^{\infty} g(t) d t$ we mean 


$$
\lim _{\epsilon \rightarrow 0}\left[\int_{-\infty}^{-\epsilon} g(t) d t+\int_{\epsilon}^{\infty} g(t) d t\right]
$$

as opposed to the definition

$$
\lim _{R \rightarrow 0}\left[\int_{-\infty}^{R} g(t) d t+\lim _{S \rightarrow 0} \int_{S}^{\infty} g(t) d t\right]
$$

where R and S approach zero independently of each other.

It can be shown that the Hilbert transform is the convolution of $x(t)$ with $1 / \pi t$, or

$$
\begin{aligned}
\widehat{x}(t) & =x(t) * \frac{1}{\pi t} \\
& =\frac{1}{\pi} P \int_{-\infty}^{\infty} \frac{x(\tau)}{t-\tau} d \tau
\end{aligned}
$$

\subsection{Properties of Fourier and Hilbert Transforms}

It can be shown that if a sequence is causal, then the real and imaginary parts of its Fourier Transform are related by a Hilbert Transform integral. We shall explore this and some other key properties of these two transforms in this section (presented without proof).

\subsubsection{Real- and Imaginary-Part Sufficiency for Causal Sequences}

Any sequence can be represented as the sum of an even sequence and an odd sequence. Letting $h_{e}(n)$ and $h_{o}(n)$ represent the even and odd part of $h(n)$, where $\mathrm{n}$ denotes the discrete time index, then

$$
h(n)=h_{e}(n)+h_{o}(n)
$$

where

$$
h_{e}(n)=\frac{1}{2}[h(n)+h(-n)]
$$

and

$$
h_{o}(n)=\frac{1}{2}[h(n)-h(-n)]
$$

If $h(n)$ is causal, then it is possible to recover $h(n)$ from $h_{e}(n)$ and to recover $\mathrm{h}(\mathrm{n})$ for $n \neq 0$ from $h_{o}(n)$. If we define

$$
u_{+}(n)= \begin{cases}2 & \text { if } n \geq 0 \\ 1 & \text { if } n=0 \\ 0 & \text { if } n<0\end{cases}
$$


then we can write

$$
h(n)=h_{e}(n) u_{+}(n)
$$

and

$$
h(n)=h_{o}(n) u_{+}(n)+h(0) \delta(n)
$$

where $\delta(n)$ is the Kronecker delta function [1].

\subsubsection{Definitions of Temporal Frequency}

For clarity, let us define the term "frequency" in its various forms. The standard definition of temporal frequency $f$ in units of Hertz $(\mathrm{Hz})$ is "cycles per unit of time," or "cycles per second." It is often useful to define radian (or angular) frequency $\Omega$ in units of radians per second, where:

$$
\Omega=2 \pi f \quad \text { (radians per second) }
$$

In digital signal processing, we often wish to calculate the Continuous Fourier Transform of a discrete-time sequence. In this case, we often use an angular "frequency. " Let $T$ be the sampling period in seconds, where the sampling frequency $f_{s}=1 / T$. Then, for a discrete-time signal, we can define its continuous-time angular "frequency" (or angle) in units of radians as

$$
\omega=\Omega T \quad \text { (radians) }
$$

\subsubsection{Relationship Between the Fourier and Hilbert Transforms}

As a consequence of the real-and imaginary part sufficiency properties of the Fourier Transform, we can show the following: If the discrete-time signal $h(n)$ is real, causal and stable, then its Continuous Fourier Transform

$$
H\left(e^{j \omega}\right)=H_{R}\left(e^{j \omega}\right)+j H_{I}\left(e^{j \omega}\right)
$$

is completely known if we know either the real part $H_{R}\left(e^{j \omega}\right)$ or the imaginary part $H_{I}\left(e^{j \omega}\right)$ and $h(0)$. This is because $H_{R}\left(e^{j \omega}\right)$ is the Fourier Transform of $h_{e}(n)$ and $j H_{I}\left(e^{j \omega}\right)$ is the Fourer Transform of $h_{o}(n)$.

Earlier, we said that if a sequence is causal, then the real and imaginary parts of its Fourier Transform are related by a Hilbert Transform integral. The following equations give that Hilbert Transform relationship [1].

$$
\begin{gathered}
H_{I}\left(e^{j \omega}\right)=\frac{1}{2 \pi} P \int_{-\pi}^{\pi} H_{R}\left(e^{j \omega}\right) \cot \left(\frac{\theta-\omega}{2}\right) d \theta \\
H_{R}\left(e^{j \omega}\right)=h(0)-\frac{1}{2 \pi} P \int_{-\pi}^{\pi} H_{I}\left(e^{j \omega}\right) \cot \left(\frac{\theta-\omega}{2}\right) d \theta
\end{gathered}
$$

\subsubsection{The Minimum Phase Condition}

Consider a z-Transform $H(z)$ of a sequence $h(n)$ written in polar (or phasor) form:

$$
h(n) \stackrel{Z}{\longleftrightarrow} H(z)=|H(z)| e^{j \arg [H(z)]}
$$


Now consider the consequences of taking the complex logarithm of $H(z)$, defined as follows:

$$
\begin{aligned}
\widehat{h}(n) \stackrel{z}{\longleftrightarrow} \widehat{H}(z) & =\log [H(z)] \\
& =\log |H(z)|+j \arg [H(z)] \\
& =H_{R}(z)+j H_{I}(z)
\end{aligned}
$$

We know that $H\left(e^{j \omega}\right)=\left.H(z)\right|_{z=e^{j \omega}}$. The sequence $\widehat{h}(n)$ is commonly known as the complex cepstrum of $h(n)$ [1]. The use of the complex logarithm in the cepstrum forms the basis of why we call such processing homeomorphic (or homomorphic), because the transformation is a homeomorphism [1,25].

If we assume $\widehat{H}(z)$ to be the z-transform of a real, causal, stable sequence $\widehat{h}(n)$, and assume that $\widehat{H}(z)$ is analytic in a region that includes the unit circle, then the results of the last section imply that $\log |H(z)|$ and $\arg [H(z)]$ are Hilbert transforms of each other, as given in the following two equations [1].

$$
\begin{gathered}
\arg H\left(e^{j \omega}\right)=\frac{1}{2 \pi} P \int_{-\pi}^{\pi} \log \left|H\left(e^{j \omega}\right)\right| \cot \left(\frac{\theta-\omega}{2}\right) d \theta \\
\log \left|H\left(e^{j \omega}\right)\right|=h(0)-\frac{1}{2 \pi} P \int_{-\pi}^{\pi} \arg \left[H\left(e^{j \omega}\right)\right] \cot \left(\frac{\theta-\omega}{2}\right) d \theta
\end{gathered}
$$

The requirement that $\log |H(z)|$ and $\arg [H(z)]$ are Hilbert transforms of each other is known as the minimum phase condition. This corresponds to the condition that $\widehat{h}(n)$ is causal. This also means that $\widehat{H}(z)$ must be analytic everywhere outside the unit circle [27]. Because $\widehat{H}(z)=\log [H(z)]$, this requires that there be no poles or zeros of $H(z)$ outside the unit circle. An equivalent condition is that there exist a causal, stable inverse system with system function $H^{-1}(z)$ such that $H(z) H^{-1}(z)=1$.

We can summarize three equivalent expressions of the minimum phase condition as follows:

- $\log |H(z)|$ and $\arg [H(z)]$ are Hilbert transforms of each other.

- $H(z)$ has no poles or zeros outside the unit circle.

- There exists a causal, stable inverse system with system function $H^{-1}(z)$ such that $H(z) H^{-1}(z)=1$.

\subsection{Discrete Fourier Transform Properties and the Hilbert Transform}

In the previous sections, we discussed Continuous Fourier Transforms (CFT's) of discrete sequences. For implementation on a computer, we must use Discrete Fourier Transforms (DFT's). Therefore, let us examine the properties described above in terms of finite-length periodic signals and DFT's.

Similar to the discussion above for infinite-length sequences, any finite-length periodic sequence $\tilde{h}(n)$ can be represented as the sum of an even sequence and an odd sequence. If we let $\tilde{h}_{e}(n)$ and $\tilde{h}_{o}(n)$ represent the even and odd parts of $\tilde{h}(n)$, where $\mathrm{n}$ denotes the discrete time index, then we can write

$$
\tilde{h}(n)=\tilde{h}_{e}(n)+\tilde{h}_{o}(n), \quad n=0,1,2, \ldots, N-1
$$

where

$$
\tilde{h}_{e}(n)=\frac{1}{2}[\tilde{h}(n)+\tilde{h}(-n)], \quad n=0,1,2, \ldots, N-1
$$

and

$$
\tilde{h}_{o}(n)=\frac{1}{2}[\tilde{h}(n)-\tilde{h}(-n)], \quad n=0,1,2, \ldots, N-1
$$


Let us now define a window $\tilde{u}_{N}(n)$ as a periodic sequence

$$
\tilde{u}_{N}(n)= \begin{cases}1, & n=0, N / 2 \\ 2, & n=1,2, \ldots,(N / 2)-1 \\ 0, & n=(N / 2+1), \ldots, N-1\end{cases}
$$

From the analyses above, it can be shown that for $N$ even, we can express $\tilde{h}(n)$ as

$$
\tilde{h}(n)=\tilde{h}_{e}(n) \tilde{u}_{N}(n)
$$

and

$$
\tilde{h}(n)=\tilde{h}_{o}(n) \tilde{u}_{N}(n)+h(0) \delta(n)+h\left(\frac{N}{2}\right) \delta\left(n-\frac{N}{2}\right)
$$

Note that given the even part $\tilde{h}_{e}(n)$, we can recover $\tilde{h}(n)$ completely. However, because $\tilde{h}_{o}(n)$ is always zero at $n=0$ and $n=N / 2, \tilde{h}(n)$ can be recovered from $\tilde{h}_{o}(n)$ only when $n \neq N / 2$.

Earlier, using the real-part sufficiency property of the z-transform, we were able to relate the log magnitude to the phase when a sequence is minimum phase. However, for the Discrete Fourier Transform, it is not possible in general to develop a parallel relationship by which the log magnitude and the phase of the DFT are related [1].

The reason for this is that for finite length sequences $h(n)$ such as the ones we discussed above, the z-transform has only zeros (no poles). However, the log of the transform $H(z)$ has singularities corresponding to both the poles and zeros of $H(z)$. The inverse transform of such a function is of infinite duration. The DFT assumes finite length periodic sequences in both the time and frequency domains, so the inverse transform of the log of the transform cannot in general be represented by the Discrete Fourier Transform.

However, for real-world signals, if we are careful about choosing the size of $\mathrm{N}$ to avoid time domain aliasing, we can obtain a very useful approximation that yields good results. We can write the relationship between the real and imaginary parts of the transform in terms of DFT's and circular convolutions as follows, assuming finite length, periodic sequences in both the time- and frequency domains [1]:

$$
\begin{gathered}
j H_{I}(k)= \begin{cases}\frac{1}{N} \sum_{m=0}^{N-1} H_{R}(m) V_{N}((k-m))_{N}, & 0 \leqslant k \leqslant N-1 \\
0 & \text { otherwise }\end{cases} \\
H_{R}(k)= \begin{cases}\frac{1}{N} \sum_{m=0}^{N-1} j H_{I}(m) V_{N}((k-m))_{N}+h(0)+(-1)^{k} h(N / 2), & 0 \leqslant k \leqslant N-1 \\
0 & \text { otherwise }\end{cases} \\
V_{N}(k)= \begin{cases}-j 2 \cot (\pi k / N), & 0<k<N-1, k \text { odd } \\
0 & \text { otherwise }\end{cases}
\end{gathered}
$$

Where $\mathrm{k}$ denotes the discrete frequency index, $k=0,1,2, \ldots, N-1$ and $((\cdot))_{N}$ denotes the quantity inside the double brackets modulo $N$ [1]. 


\subsection{Realization of the Complex Cepstrum Using the Complex Logarithm}

We can exploit the process described above to build an algorithm to solve the following problem: Given a finitelength, real, causal, stable sequence $h(n)$, construct a realization of the complex cepstrum $\widehat{h}(n)$ of that signal using the complex logarithm.

Assume that $H(z)$ is the z-transform of a finite-length sequence $h(n)$. If $H(z)$ has no zeros outside the unit circle, then we know from the discussion above that given only $\log \left|H\left(e^{j \omega}\right)\right|$ we can compute $\arg \left[H\left(e^{j \omega}\right)\right]$.

If we calculate $\widehat{H}(z)=\log [H(z)]$, it will correspond to a causal sequence $\widehat{h}(n)$ which in general, will have infinite duration. So, if we use this scheme with a DFT, we must truncate the generally infinite sequence. Note that by definition, $\widehat{h}(n)$ is the complex cepstrum of $h(n)[1]$.

We know that the DFT of the length $\mathrm{N}$ sequence $h(n)$ can be written

$$
\begin{aligned}
H(k) & =\sum_{n=0}^{N-1} h(n) e^{j \frac{2 \pi}{N} k n} \\
& =\left.H(z)\right|_{z=e^{j(2 \pi k / N)}} \quad k=0,1,2, \ldots, N-1
\end{aligned}
$$

where $\mathrm{N}$ must be chosen to have at least the length of the sequence $\mathrm{h}(\mathrm{n})$ and $\mathrm{k}$ is the discrete frequency index $k=0,1,2, \ldots, N-1$.

Using the notion of the complex cepstrum from above, consider the DFT $\widehat{H}_{p}(k)$ defined by the following, where the subscript $p$ emphasizes the fact that the DFT is periodic:

$$
\begin{aligned}
\widehat{H}_{p}(k) & =\log [H(k)] \\
& =\widehat{H}_{R}(k)+j \widehat{H}_{I}(k) \\
& =\log |H(k)|+j \operatorname{jarg}[H(k)]
\end{aligned}
$$

We know from the periodic nature of the DFT, that $\widehat{H}_{p}(k)$ corresponds to a time domain aliased periodic infinite length sequence $\widehat{h}_{p}(n)$ which is the complex periodic cepstrum of $h(n)$. Therefore, if we compute $\widehat{h}_{p}(n)$ using the inverse DFT, we must choose $\mathrm{N}$ to be at least as long as the sequence $h(n)$ to avoid time domain aliasing. The quantity $\widehat{h}_{p}(n)$ can be written as follows:

$$
\begin{aligned}
\widehat{h}_{p}(n) & =\sum_{r=-\infty}^{\infty} \widehat{h}(n+r N) \\
& =\text { time aliased version of } \widehat{h}(n) \\
& \approx \widehat{h}(n) \text { for } \mathrm{N} \text { large }
\end{aligned}
$$

where $r$ is an integer. Note that to complete this calculation, we must also carefully unwrap the phase. Efficient methods for calculating the complex cepstrum and unwrapping its phase are described in $[1,7]$. Implementations of this calculation are given in FORTRAN [7] and in the MATLAB command "cceps" [28].

\subsection{Minimum Phase Realization Given a Finite-Length Sequence}

Consider the following problem: Given a finite-length, real, causal, stable sequence $h(n)$, construct a minimum phase realization $\widehat{h}(n)$ of $\mathrm{h}(\mathrm{n})$.

This is not the phase retrieval problem we eventually wish to solve, but it gives us some extremely important insights into some key issues, and suggests a direction toward the solution we desire. Special consideration is given 
to the fact that for computer implementation with a DFT we must use finite-length sequences and assume that they are periodic. We adapt the theory of infinite length sequences for use with finite-length sequences.

First, let us compute the DFT $H(k)$ of $h(n)$ and the spectral modulus $|H(k)|$. Next calculate the logarithm of the DFT modulus $|H(k)|$. From Equation (2.46) in the last section, we see that this result will correspond to the real part $\widehat{H}_{R}(k)$ of $\widehat{H}_{p}(k)$. We write this as follows:

$$
\widehat{H}_{R}(k)=\log |H(k)|
$$

From $\widehat{H}_{R}(k)$ we can compute the periodic real cepstrum $c_{p}(n)$ as follows:

$$
\begin{aligned}
c_{p}(n) & =\operatorname{IDFT}\left\{\widehat{H}_{R}(k)\right\} \\
& =\sum_{r=-\infty}^{\infty} c(n+r N) \\
& =\text { Aliased Even Part of } \widehat{h}(n)
\end{aligned}
$$

Note that $c_{p}(n)$ is a periodic, time-aliased version of the real cepstrum $c(n)$. Similar to the analysis in the last section, we can compute the periodic complex cepstrum $\widehat{h}_{c p}(n)$ from the periodic real cepstrum $c_{p}(n)$. We can write

$$
\begin{aligned}
\widehat{h}_{c p}(n)= & \begin{cases}c_{p}(n), & n=0, N / 2 \\
2 c_{p}(n), & n=1,2, \ldots,(N / 2)-1 \\
0, & n=(N / 2+1), \ldots, N-1\end{cases} \\
& =c_{p}(n) \tilde{u}_{N}(n) \\
& =\left[\sum_{r=-\infty}^{\infty} c(n+r N)\right] \tilde{u}_{N}(n) \\
& =[\text { Aliased Even Part of } \widehat{h}(n)] \tilde{u}_{N}(n) \\
& \approx \widehat{h}(n), \text { for large } \mathrm{N}
\end{aligned}
$$

where $\widehat{h}(n)$ is the complex cepstrum of $h(n)$. Comparing (2.49) and (2.56), we see that

$$
\widehat{h}_{c p}(n) \neq \widehat{h}_{p}(n)
$$

because in $\widehat{h}_{p}(n)$ the even part of $\widehat{h}(n)$ is aliased, rather than $\widehat{h}(n)$ itself. Nonetheless, in Equation (2.56) we claim that the periodic complex cepstrum $\widehat{h}_{c p}(n)$ is an approximation to $\widehat{h}(n)$ when the number of samples $N$ is large. Let us explore the justification for this assertion.

From the properties of the complex cepstrum discussed earlier, we saw in property $P 4$ that if $h(n)$ is of finite duration, then $\widehat{h}(n)$ will nevertheless have infinite duration. In spite of property $P 4$, it can be shown that for an input sequence of length $\mathrm{N}$, we need only $\mathrm{N}$ samples of $\widehat{h}(n)$ to determine $x(n)$ [1].

From Property P1, we note that in general, $\widehat{h}(n)$ decays faster than an exponential sequence, so it is expected that the approximation $\widehat{h}_{c p}(n) \approx \widehat{h}(n)$ would become increasingly better as $\mathrm{N}$ becomes larger. This means that for real-world signals, we may need to take steps to ensure that the complex logarithm is sampled at a high enough rate to avoid severe aliasing in the computation of the complex cepstrum. For example, (1) If we are given a sequence $h(n)$ and wish to construct a minimum phase realization, then we may need to append zeros to $h(n)$ so that the effective DFT $H(k)$ is interpolated. (2) If we are given $\widehat{H}(k)$ and wish to construct a minimum phase realization of $h(n)$ or reconstruct the phase, we may need to increase $N$ in the frequency domain (make the frequency domain sampling interval $\Delta f$ smaller) by interpolating the spectrum using other means [28]. 
Figure (2.1) shows a block diagram of the algorithm for calculating a minimum phase realization of $\widehat{h}(n)$ given a finite-length, real, causal, stable sequence $h(n)$. Note that implementations of this calculation are available in FORTRAN [7] and in the MATLAB command "rceps" [28]. 


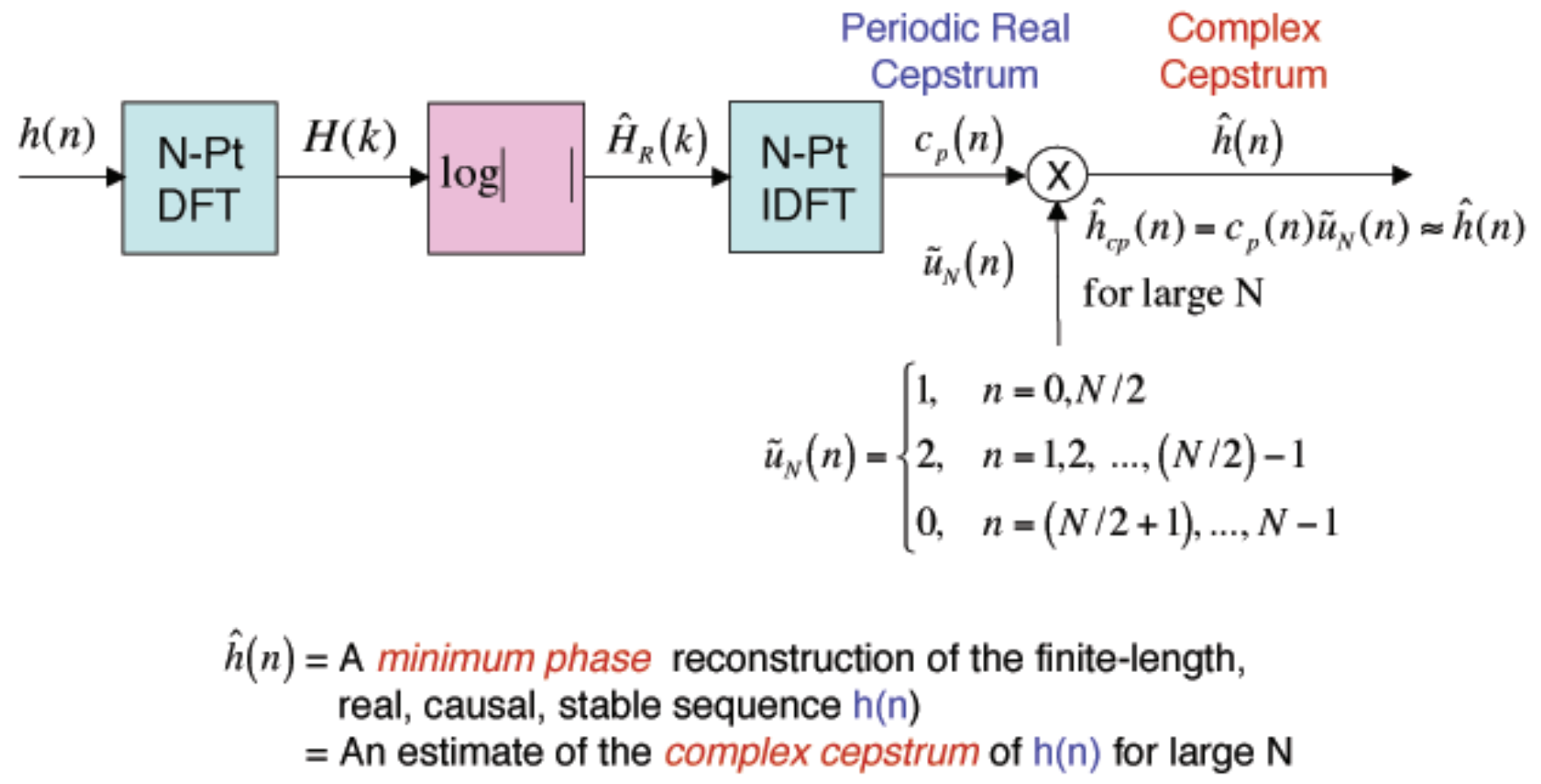

Figure 2.1: Block Diagram of the Minimum Phase Reconstruction Algorithm. Given a finite-length, real causal, stable sequence $h(n)$, we can construct a minimum phase realization $\widehat{h}(n)$ of $h(n)$. The signal $\widehat{h}(n)$ is an estimate of the complex cepstrum of $h(n)$. 


\section{Chapter 3}

\section{Phase Reconstruction Given Only the Spectral Modulus}

Using the results of the last chapter, we show that if we are given only the modulus $|\widehat{H}(k)|$ (as we are in the Lightning Protection Program), we can reconstruct a minimum phase sequence $\widehat{h}(n)$ and recover its phase from the imaginary part of its DFT. The algorithm is summarized and depicted in Figure (3.1).

Let us now consider the following problem: Given only the modulus of a DFT, reconstruct the phase of the DFT. We can use some of the results from the last section and expand on them to build a reconstruction algorithm.

\subsubsection{Construct an Estimate of the Minimum Phase Realization Given Only the Spectral Modulus}

First, calculate the logarithm of the given DFT modulus $|H(k)|$. From the earlier discussion of Equation (2.46), we see that this result will correspond to the real part $\widehat{H}_{R}(k)$ of $\widehat{H}_{p}(k)$. We write this as follows:

$$
\widehat{H}_{R}(k)=\log |H(k)|
$$

From $\widehat{H}_{R}(k)$ we can compute the periodic real cepstrum $c_{p}(n)$ as follows:

$$
\begin{aligned}
c_{p}(n) & =\operatorname{IDFT}\left\{\widehat{H}_{R}(k)\right\} \\
& =\sum_{r=-\infty}^{\infty} c(n+r N) \\
& =\text { Aliased Even Part of } \widehat{h}(n)
\end{aligned}
$$

Note that $c_{p}(n)$ is a periodic, time-aliased version of the real cepstrum $c(n)$. Similar to the analysis in the last section, we can compute the periodic complex cepstrum $\widehat{h}_{c p}(n)$ from the periodic real cepstrum $c_{p}(n)$. We can 
write

$$
\begin{aligned}
\widehat{h}_{c p}(n)= & \begin{cases}c_{p}(n), & n=0, N / 2 \\
2 c_{p}(n), & n=1,2, \ldots,(N / 2)-1 \\
0, & n=(N / 2+1), \ldots, N-1\end{cases} \\
& =c_{p}(n) \tilde{u}_{N}(n) \\
& =\left[\sum_{r=-\infty}^{\infty} c(n+r N)\right] \tilde{u}_{N}(n) \\
& =[\text { Aliased Even Part of } \widehat{h}(n)] \tilde{u}_{N}(n) \\
& \approx \widehat{h}(n), \text { for large } \mathrm{N}
\end{aligned}
$$

where $\widehat{h}(n)$ is the complex cepstrum of $h(n)$. Comparing Equation (2.49) and Equation (3.5), we see that

$$
\widehat{h}_{c p}(n) \neq \widehat{h}_{p}(n)
$$

because in $\widehat{h}_{p}(n)$ the even part of $\widehat{h}(n)$ is aliased, rather than $\widehat{h}(n)$ itself. Nonetheless, in Equation (3.5) we claim that the periodic complex cepstrum $\widehat{h}_{c p}(n)$ is an approximation to $\widehat{h}(n)$ when the number of samples $N$ is large. In the previous section, we showed that the approximation for large $\mathrm{N}$ is theoretically justified. Later in this report, we demonstrate with simulated signals that this approximation is justified in practice and very useful.

\subsubsection{Reconstruct the Phase from the Estimated Complex Cepstrum}

Now, if we wish to reconstruct the phase, we need only compute the DFT of $\widehat{h}(n)$ and save its imaginary part because we know that $\widehat{H}(k)$ provides a discrete-time estimate of the log of the continuous time Fourier transform as follows:

$$
\begin{aligned}
\widehat{H}(k) & =\log [H(k)] \\
& =\log |H(k)|+\operatorname{jarg}[H(k)]
\end{aligned}
$$

Saving the imaginary part of $\widehat{H}(k)$ gives us the reconstructed phase.

$$
\arg [H(k)]=\operatorname{Im}\{\widehat{H}(k)\}
$$

This algorithm is depicted in block diagram form in Figure (3.1).

\subsection{Relationship with the Minimum Phase Realization Algorithm}

The minimum phase realization algorithm is depicted in block diagram form in Figure (2.1). Comparing Figure Figure (3.1) and Figure (2.1), we see that they contain many of the same elements.

\subsection{Limitations of the Algorithm}

Every algorithm has limitations and tradeoffs. When using this phase retrieval algorithm, the following points must be kept in mind. (1) The algorithm is not designed to be used with noisy signals. We show later that the 


$$
\hat{h}_{c p}(n)=c_{p}(n) \tilde{u}_{N}(n) \approx \hat{h}(n) \text { for large } \mathrm{N}
$$

\section{Input Spectral Modulus}

$$
\begin{gathered}
\text { Periodic Real Complex } \\
\text { Cepstrum Cepstrum }
\end{gathered} \hat{H}(k)=\log |H(k)|+j \arg [H(k)]
$$
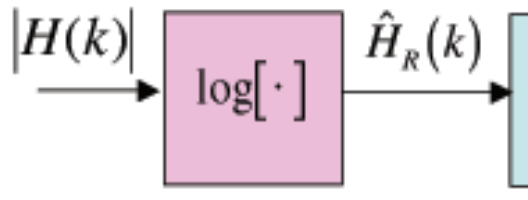

$$
\begin{aligned}
& \begin{array}{l}
\text { N-Pt } \\
\text { IDFT }
\end{array} \stackrel{c_{p}(n)}{\longrightarrow} \stackrel{\hat{h}(n)}{\longrightarrow} \begin{array}{l}
\mathrm{N}-\mathrm{Pt} \\
\mathrm{DFT}
\end{array} \\
& \tilde{u}_{N}(n)= \begin{cases}1, & n=0, N / 2 \\
2, & n=1,2, \ldots,(N / 2)-1 \\
0, & n=(N / 2+1), \ldots, N-1\end{cases}
\end{aligned}
$$
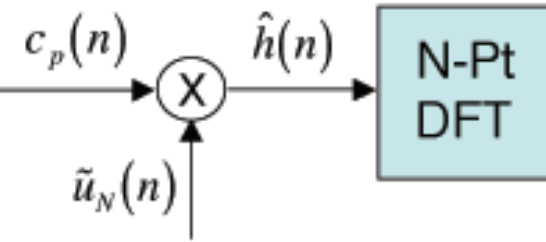

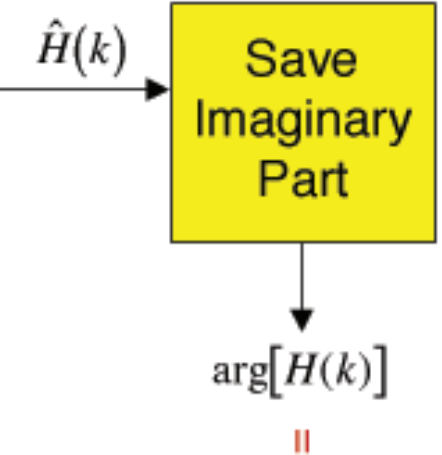

Reconstructed Phase of $h(n)$

$$
\begin{aligned}
\hat{h}(n)= & \text { A minimum phase reconstruction of a finite-length, } \\
& \text { real, causal, stable sequence } \mathrm{h}(\mathrm{n}) \text { corresponding } \\
& \text { to the measured input spectral modulus }|\mathrm{H}(\mathrm{k})| \\
= & \text { An estimate of the complex cepstrum of } \mathrm{h}(\mathrm{n}) \text { for large } \mathrm{N}
\end{aligned}
$$

Figure 3.1: Block Diagram of the proposed Phase Reconstruction Algorithm. Given the input spectral modulus $|H(k)|$, we can construct a finite-length, real, causal, stable sequence $\widehat{h}(n)$ corresponding to that spectral modulus. The signal $\widehat{h}(n)$ is a minimum phase reconstruction of $h(n)$, and it is an estimate of the complex cepstrum of $h(n)$. We can retrieve the phase of $H(k)$ by saving the imaginary part of the DFT of $\widehat{h}(n)$. 
algorithm can be fairly robust to noise effects, but more study is needed to determine its efficacy for any given

application. (2) Regardless of the properties of the actual signal $h(n)$, the reconstruction $\widehat{h}(n)$ obtained from the algorithm is constrained to have the minimum phase property. For some systems (mostly theoretical ones), this would not be appropriate. However, for most practical experimental applications of interest, the minimum phase assumption is very reasonable. This is the case for the lightning protection application. The measurements are real, causal and stable. (3) The number of samples in the signals $(N)$ must be even. This requirement is easy to meet in practice, and is likely to be met naturally. This is because most signal processing systems are designed to use Fast Fourier Transforms (FFT's), which are fast only when the FFT size is a power of two $\left(N=2^{r}\right)$ where $r$ is an integer. One can easily adjust the signal length and FFT size by a variety of means, including adjustments in the data acquisition system and zero padding. (4) The length of the signals must be large compared with the nonzero part of the measurements to avoid cepstral aliasing. Again, this length is easily adjustable using the data acquisition system and/or zero padding.

\subsection{MATLAB Implementation}

The author has written a MATLAB M-file named "Phase_Retrieval_GAC.m" that implements the phase retrieval algorithm and the simulations shown in this report. The code simulates an appropriate signal, asks the user to specify the desired signal-to-noise ratio (SNR), then reconstructs the phase and plots the results.

To facilitate understanding, the plots in this report have labels that use the variable names use in the M-file Phase_Retrieval_GAC.m. This M-file can be easily edited to create a code for use in a Lightning Protection project. A function called Build_W_V_GAC_New.m is called by the main code to simulate additive white Gaussian noise (WGN). The two M-files are available by contacting the author. 


\section{Chapter 4}

\section{Simulation Experiments}

In this section, we examine the efficacy of the phase reconstruction algorithm by testing it with simulated signals, both noiseless and noisy. We use three simulated signals; A noiseless signal, a signal containing moderate additive noise, and a signal containing heavy additive noise.

\subsection{Experimental Approach}

In this report, we do not embark upon a full analysis of noise effects on reconstruction performance. Such an analysis is left for future work. The goal here is to obtain a rough idea of the robustness of the algorithm to noisy measurements to assess whether or not it is reasonable to proceed with further work with real-world measurements. To this end, we simply simulate noisy measured spectral moduli, reconstruct minimum-phase realizations, and use visual inspection of plots of those realizations to make an engineering judgment as to robustness. Future work should include a statistical error analysis.

\subsection{Definitions for the Simulations}

We define three simulated signals; A noiseless signal, a signal containing moderate additive noise, and a signal containing heavy additive noise. The noiseless case demonstrates the theory as developed above. The noisy signals are examined because for real-world applications, there is generally some noise included in the signals. For the Lightning Protection applications the measured moduli contain a small amount of noise. The simulations here contain more noise than that observed in the Lightning Protection measurements. In this analysis, we wish to examine the robustness of the algorithm to moderate and heavy noise so we can estimate whether the algorithm might be useful for general Lightning Protection work. A full analysis of this possibility with real signals should be carried out in future work.

\subsubsection{Define the Noiseless Simulated Signal}

The simulated signals are designed to meet the requirements of the algorithm's assumptions. Let

$$
x(n)=\alpha^{n} u(n)
$$

where we have assumed that the sampling period $T$ is unity, and $u(n)$ is the unit step or Heaviside function $[1,4,6]$. We assume that $\alpha$ is a real constant and $|\alpha|<1$. This signal is causal because of the unit step function. 
The z-Transform of $h(n)$ is given by

$$
X(z)=\frac{1}{1-\alpha z^{-1}}, \quad|\alpha|<1
$$

$\mathrm{H}(\mathrm{z})$ has one zero at $z=0$ and one pole at $z=\alpha$. Because $|\alpha|<1$, all of the poles and zeros lie inside the unit circle. This means that the signal $x(n)$ satisfies the minimum phase condition. We see that all of the requirements of the phase reconstruction algorithm are met. The signal $x(n)$ is real, causal, and stable. In addition the minimum phase condition is useful, as we shall demonstrate.

For the noiseless case, we assume that the signal we wish to reconstruct is $x(n)$ as defined above. Using the notation of the last chapter, the reconstructed signal $\widehat{h}(n)$ should be a close estimate of $x(n)$.

To simulate the problem of phase reconstruction from modulus, we compute $|X(k)|$ and assume that this is the only knowledge we are given from our measurements. This simulates the case we encounter in the Lightning Protection problem.

\subsubsection{The Simulated Lightning Signal}

Actual lightning signals have extremely high amplitudes and energies, making them difficult to measure and process directly. In the lightning protection literature it is typical to simulate a scaled down lightning waveform and use that in studies of buildings [24]. The model used for simulating a lightning current signal is typically a double exponential of the following form:

$$
I(t)=I_{0}\left[-e^{-\beta t}+e^{-\gamma t}\right]
$$

where $I_{0}$ is a constant initial current, and $\beta$ and $\gamma$ are time constants with size about three orders of magnitude apart. A typical set of parameters used is $\beta=1 . e 4 / \mathrm{sec}$ and $\gamma=1 . e 7 / \mathrm{sec}$. If we discretize the time using the time index $n$, we can write the lightning current as follows:

$$
I(n)=I_{0}\left[-e^{-\beta n}+e^{-\gamma n}\right]
$$

If we let $a_{1}=e^{-\beta}$ and $a_{2}=e^{-\gamma}$ and use the unit step function $u(t)$ to enforce causality, then the current becomes:

$$
I(n)=I_{0}\left[-a_{1}{ }^{n} u(n)+a_{2}{ }^{n} u(n)\right]
$$

This function now has a form very similar to that of the simulation signal we used above. Its z-transform can be shown to be:

$$
I(z)=I_{0}\left[-\frac{1}{1-a_{1} z^{-1}}+\frac{1}{1-a_{2} z^{-1}}\right]
$$

where the regions of convergence are $|z|>\left|a_{1}\right|$ for the first term and $|z|>\left|a_{2}\right|$ for the second term. The function is stable for $|\beta|<1$ and $|\gamma|<1$. We see by inspection that the signal is real, causal, stable and satisfies the minimum phase property when $a_{1}$ and $a_{2}$ are chosen inside the unit circle. Therefore, the signal $I(t)$ satisifies all of the requirements of the proposed phase retrieval algorithm. This result gives us confidence in the efficacy of the algorithm for the lightning protection application.

\subsubsection{Define the Simulated Noisy Signals}

Let us now assume that the measured spectrum (and thus its inverse DFT), are corrupted by white Gaussian noise (WGN) $v(n)$ having zero mean and variance $\sigma_{v}{ }^{2}$. We denote the characteristics of the noise by $v(n) \sim N\left[0, \sigma_{v}{ }^{2}\right]$. We can then write the noisy time domain signal $z(n)$ as

$$
\begin{aligned}
z(n) & =x(n)+v(n) \\
& =\alpha^{n} u(n)+v(n)
\end{aligned}
$$


where $x(n)$ is the same signal used in the noiseless case above, and $v(n) \sim N\left[0, \sigma_{v}{ }^{2}\right]$.

For the noisy case, we assume that the signal we wish to reconstruct is $x(n)$ as defined above. However, given that the measurements are noisy, we expect that the estimate of the reconstructed signal $\widehat{h}(n)$ will be degraded from that obtained in the noiseless case. Using the notation of the last chapter, we wish to assess whether or not the reconstructed signal $\widehat{h}(n)$ is a useful estimate of $x(n)$.

To simulate the problem of phase reconstruction from modulus, we compute the modulus $|Z(k)|$ of the noisy spectrum and treat it as though it is the only knowledge we have of the underlying signal. Thus, $|Z(k)|$ becomes our input measurement as depicted in Figure (3.1). We then process $|Z(k)|$ to obtain the reconstructed signal $\widehat{h}(n)$ and its reconstructed phase $\arg [H(k)]$.

\subsubsection{Define Noise and Signal-to-Noise Ratio (SNR)}

In general, we define the signal-to-noise ratio (SNR) as follows [5]:

$$
\begin{aligned}
S N R & \triangleq \frac{\text { Signal Energy }}{\text { Noise Variance }} \\
& \triangleq \frac{E_{x}}{\sigma_{v}{ }^{2}}
\end{aligned}
$$

where the energy in signal $x(n)$ is given by

$$
E_{x} \triangleq \sum_{n=n_{0}}^{n_{1}} x^{2}(n)
$$

and we calculate the energy over the time interval between time indices $n_{0}$ and $n_{1}$. We denote noise variance by $\sigma_{v}{ }^{2}$. Here, we have assumed that the discrete-time signal has time sampling interval $T=1$ for convenience. For our simulations, we compute the energy over the entire length of the data record, $n=0,1,2, \ldots, N-1$. Note that by Parseval's Theorem, we could equivalently calculate the energy in the frequency domain $[8,4]$. We can express the SNR in decibels using the following definition:

$$
\begin{array}{rlr}
\operatorname{SNR}(d b) & =10 \log _{10}\left[\frac{E_{x}}{\sigma_{v}^{2}}\right] \\
& =10 \log _{10}[R], \quad \text { where } R \triangleq E_{x} / \sigma_{v}^{2}
\end{array}
$$

So, once we are given $E_{x}$ for our particular signal, and we know our desired $S N R(d B)$, we can solve for the required noise variance. If we define $G$ as follows, then we have:

$$
\begin{aligned}
G & \triangleq S N R(d b) / 10 \\
R & =10^{G} \\
\sigma_{v}{ }^{2} & =E_{x} / R
\end{aligned}
$$

Consider an example from this simulation. Let $E_{x}=4.2632$ and the desired $S N R(d B)=40$. Then, we see that $G=4, R=10^{4}$, and $\sigma_{v}{ }^{2}=4.2532 e-4$. 

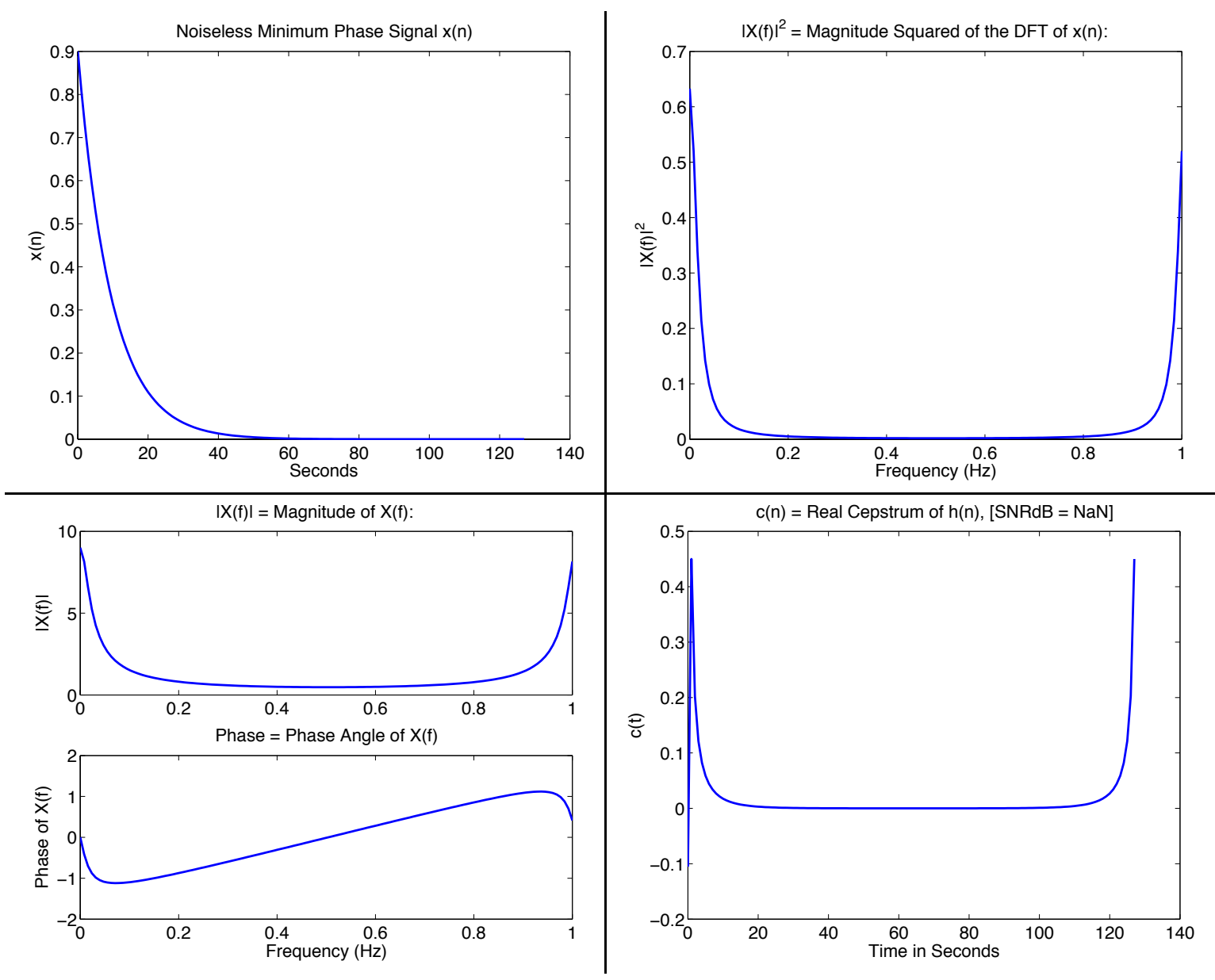

Figure 4.1: (Upper Left): The simulated noiseless minimum phase signal $x(n), N=128$. Clearly, this finitelength signal is real, causal and stable. (Upper Right): The magnitude squared of the N-point DFT of the noiseless signal $x(n)$. (Lower Left): The magnitude and phase of the N-point DFT of the noiseless signal $x(n)$. (Lower Right): $\mathrm{c}(\mathrm{n})=$ The real cepstrum $\widehat{h}(n)$ of $\mathrm{h}(\mathrm{n})$ for the noiseless case.

\subsection{Simulation Example for the Noiseless Case}

Here, we simulate the case in which the signal $x(n)$ is noiseless. Later, we add noise compare algorithm performance for the various cases. In the plot labels, we often use $f$ for frequency to indicate the frequeny in $\mathrm{Hz}$ rather than indicating the discrete frequency index $k$. Because the time sampling period $T=1$, however, the two have the same value.

The next three figures present the processing results for this experiment. They follow the processing flow in the block diagram of the last section. The figure captions are self explanatory. We see that the reconstruction $\widehat{h}(n)$ and its phase provide excellent estimates. Clearly, the algorithms performed very well for this noiseless signal. 

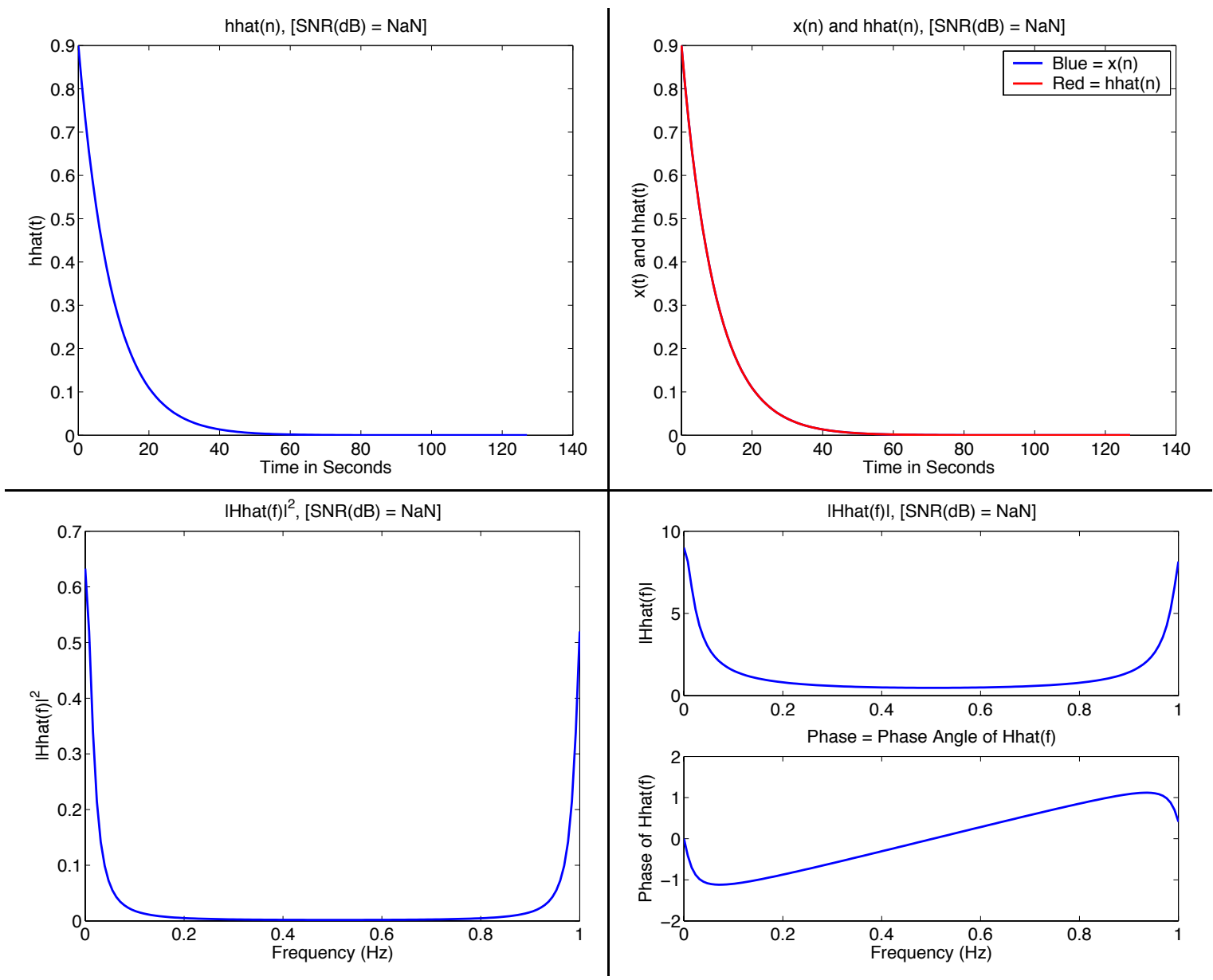

Figure 4.2: (Upper Left): The reconstructed minimum phase signal $\widehat{h}(n)$ for the noiseless case $(N=128)$. (Upper Right): The signal x(n) overlayed with the reconstructed signal $\widehat{h}(n)$ for the noiseless case. The agreement between the two is excellent $(N=128)$. (Lower Left): Magnitude squared of the DFT of the reconstructed signal $\widehat{h}(n)$ for the noiseless case $(N=128)$. (Lower Right): Magnitude $|\widehat{H}(k)|$ and phase $\arg [\widehat{H}(k)]$ for the noiseless case $(N=128)$. 

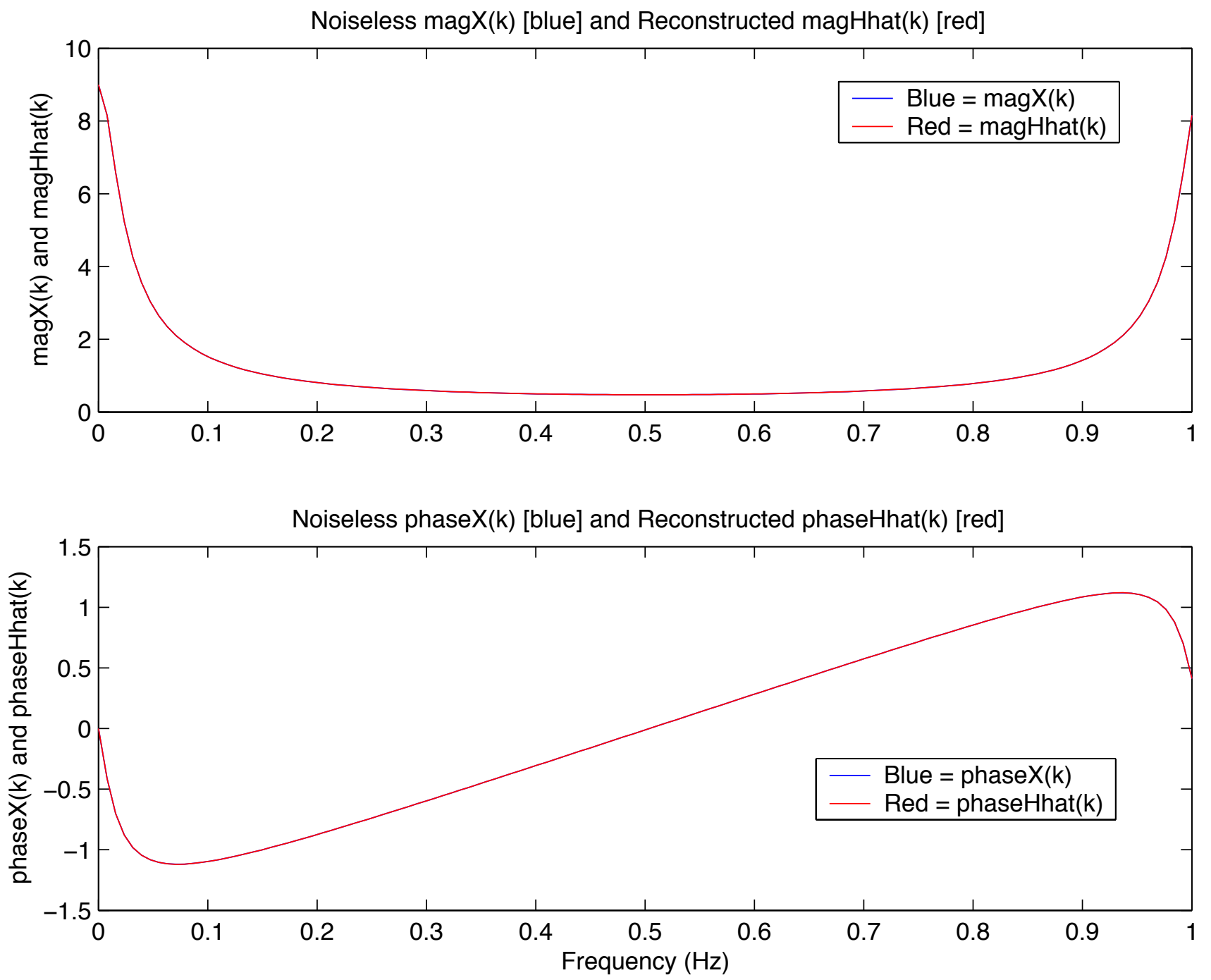

Figure 4.3: Magnitude $|X(k)|$ and phase $\arg [X(k)]$ overlayed on the reconstructed magnitude $|\widehat{H}(k)|$ and phase $\arg [\widehat{H}(k)]$ for the noiseless case $(N=128)$. The agreement is excellent. 

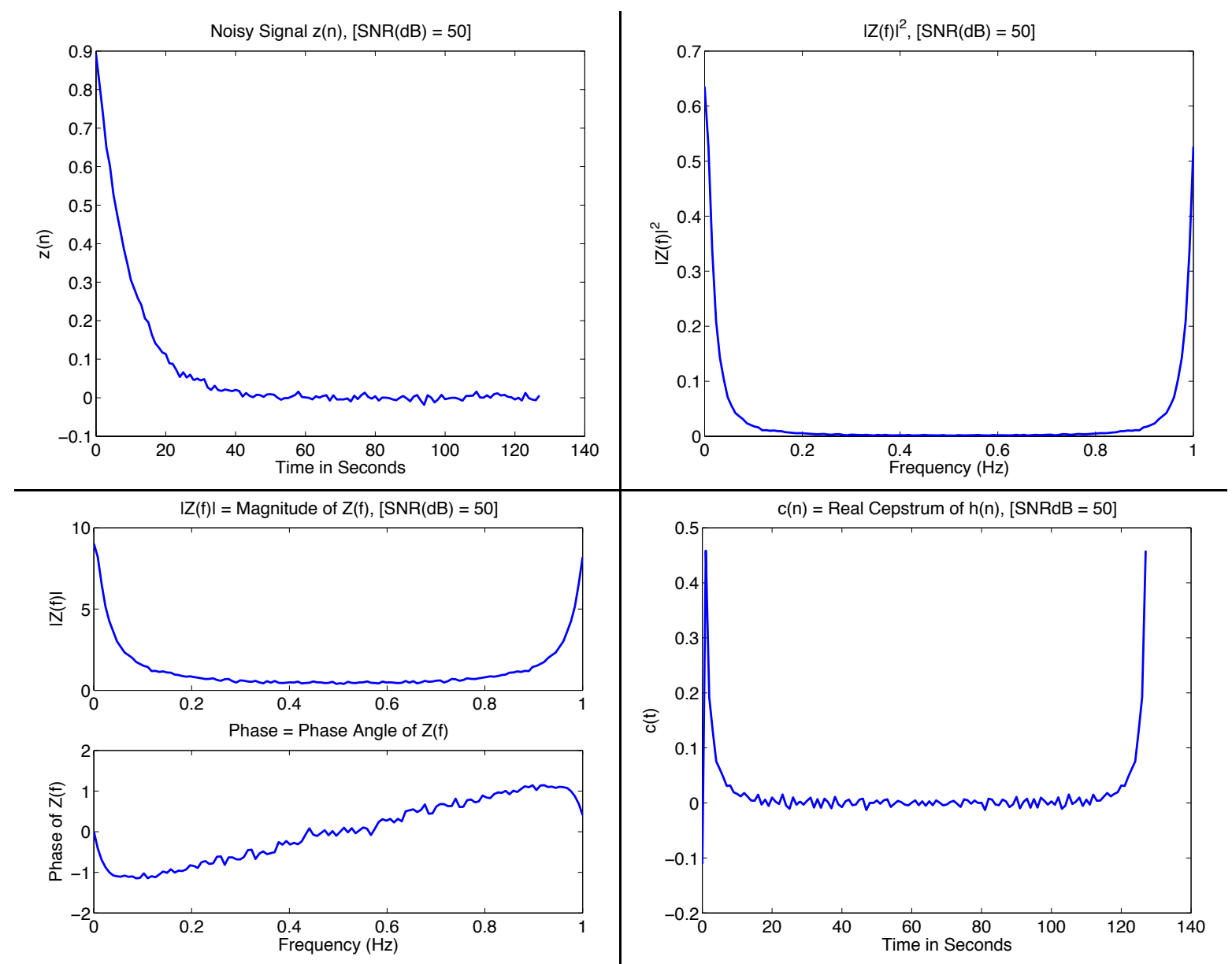

Figure 4.4: (Upper Left): The noisy minimum phase signal $z(n)$ for SNR $=1 . e 5(50 \mathrm{~dB})$. (Upper Right): The magnitude squared of the DFT of the noisy signal $\mathrm{z}(\mathrm{n})$ for $\mathrm{SNR}=50 \mathrm{~dB}$. (Lower Left): The magnitude and phase of the DFT of the noisy signal z(n) for SNR $=50 \mathrm{~dB}$. (Lower Right): $\mathrm{c}(\mathrm{n})=$ the Real Cepstrum of $\mathrm{h}(\mathrm{n})$ for $\mathrm{SNR}=$ $50 \mathrm{~dB}$.

\subsection{Simulation Example For SNR = 1.e-5 (50 dB)}

The lightning protection signals measured in the field generally have small amounts of noise [23, 22, 20, 21]. Therefore, in our first example, we wish to approximate that scenario.

We calculated the signal energy for our simulated signal and found it to be $E_{x}=4.2632$. Using the relations in the previous section, this means that if we assign the value of the noise variance to be $\sigma_{1}{ }^{2}=4.2632 . e-5$, we achieve $S N R(d B)=50 d B$, which corresponds to a magnitude ratio of 1.e5. This SNR is very reasonable for the lightning protection work. In fact, it may even be small in many situations.

The next three figures present the processing results. The figure captions are self explanatory. We see that for the reconstruction $\widehat{h}(n)$, the estimation errors are reasonably small, despite the noise. The errors in the magnitude estimate are similarly small. The errors in the phase estimate appear to be small at low frequencies. They then grow and reach a maximimum near the folding frequency. This is encouraging for the lightning protection problem, because the most important part of the spectrum lies in the low-frequency region [20, 21, 22, 23]. 

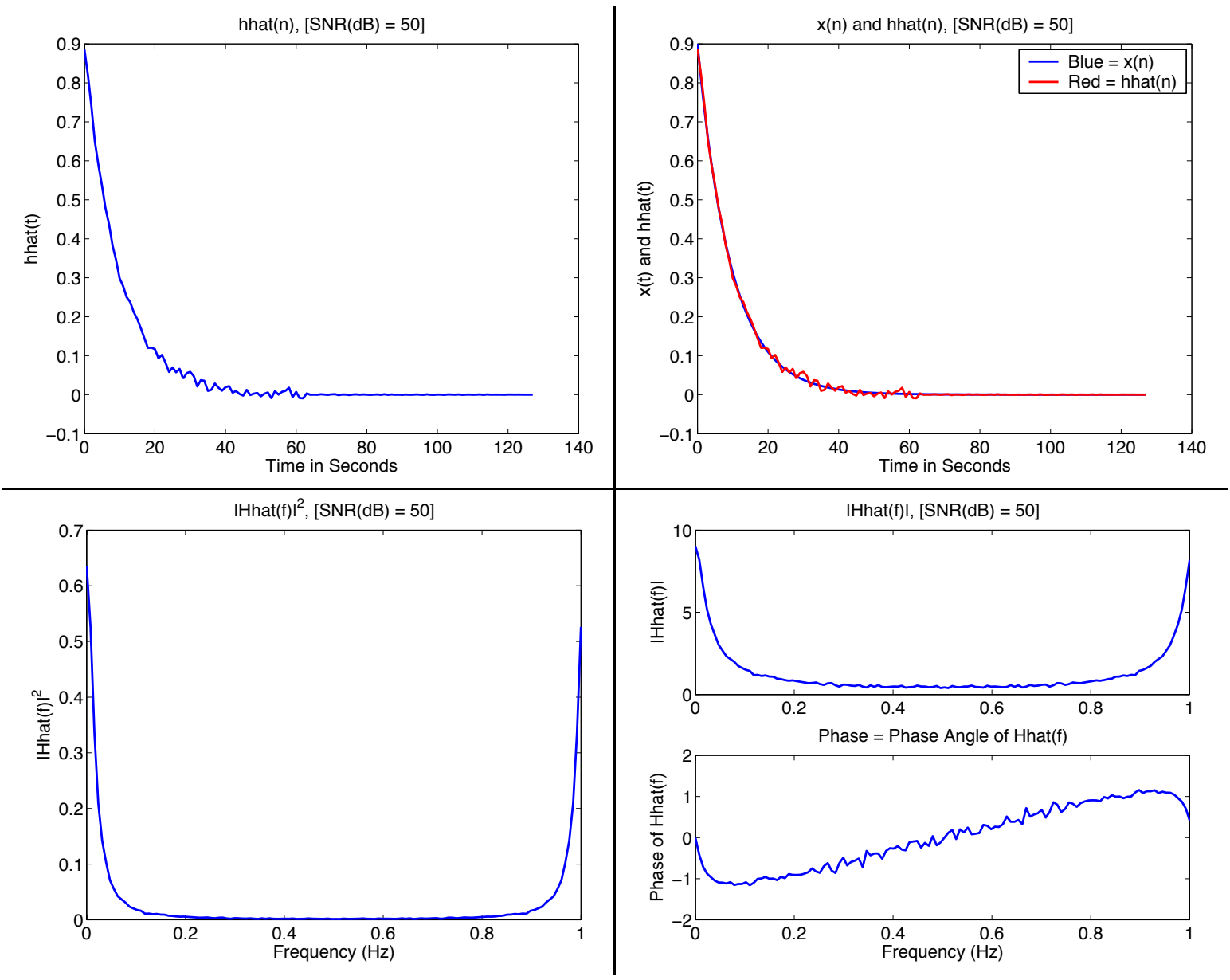

Figure 4.5: (Upper Left): The Reconstructed Minimum Phase Signal $\widehat{h}(n)$ when $\mathrm{SNR}(\mathrm{dB})=50 \mathrm{~dB}$. (Upper Right): The noiseless signal $\mathrm{x}(\mathrm{n})$ and the Reconstructed Signal $\widehat{h}(n)$ for SNR $=50 \mathrm{~dB}$. (Lower Left): The magnitude squared of the DFT of the Reconstructed Signal $\widehat{h}(n)$ for SNR $=50 \mathrm{~dB}$. (Lower Right): The magnitude and phase of the DFT of the reconstructed signal $\widehat{h}(n)$ for $\operatorname{SNR}(\mathrm{dB})=50 \mathrm{~dB}$. 

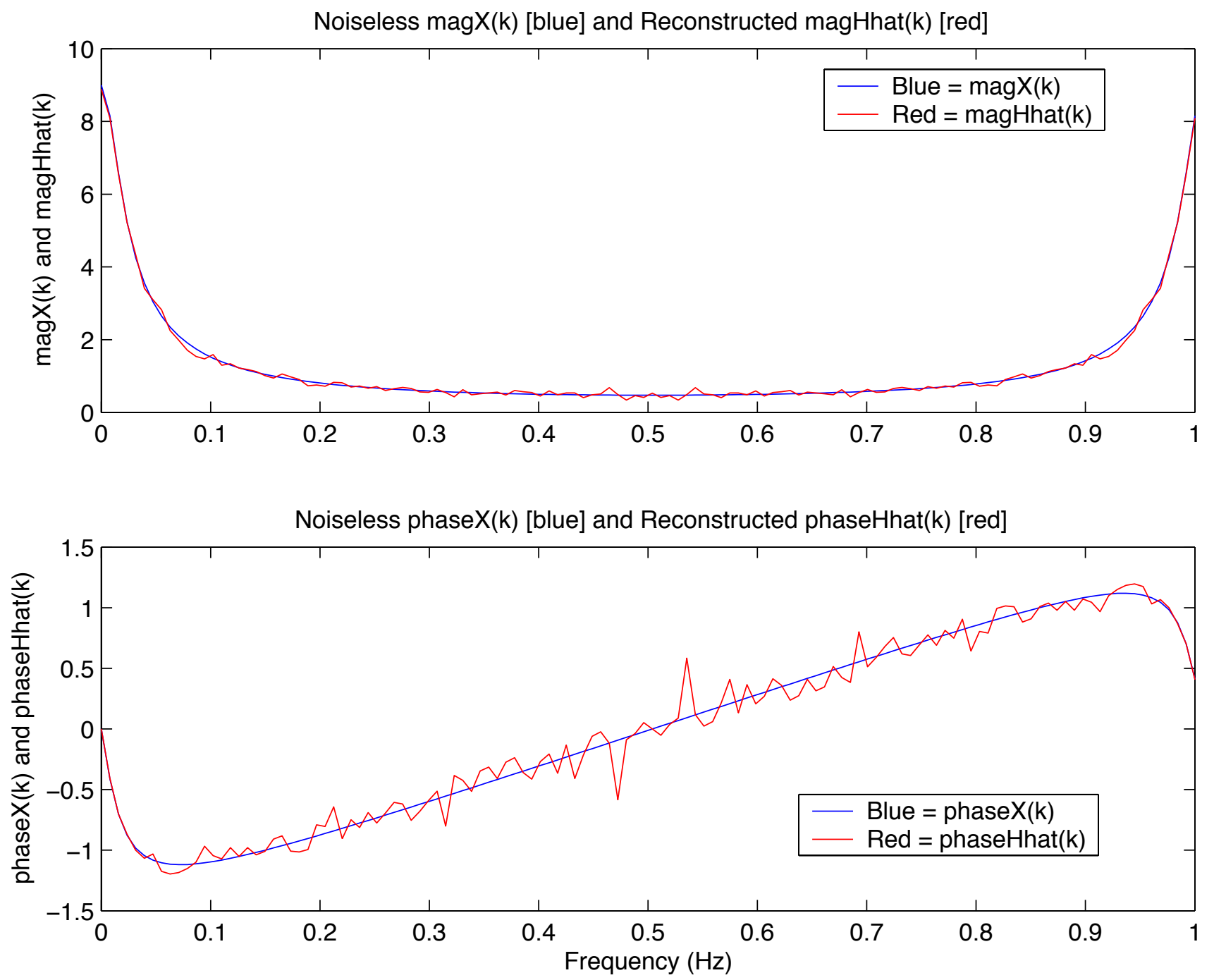

Figure 4.6: The noiseless magnitude and phase of $\mathrm{X}(\mathrm{k})$ and the reconstructed magnitude and phase of $\widehat{H}(k)$ for $\mathrm{SNR}=50 \mathrm{~dB}$. 


\subsection{Simulation Example For SNR = 1.e4 $(40 \mathrm{~dB})$}

In this example, we wish to simulate high noise measurements.

We calculated the signal energy for our simulated signal and found it to be $E_{x}=4.2632$. Using the relations in the previous section, this means that if we assign the value of the noise variance to be $\sigma_{1}{ }^{2}=4.2632 . e-4$, we achieve $S N R(d B)=40 d B$, which corresponds to a magnitude ratio of 1.e4. This SNR is much lower than that observed in the field $[23,22,20,21]$. Therefore, the results should represent at least a worst case scenario in most situations.

Figure (4.7) and Figure (4.8) present the processing results. The figure captions are self explanatory. The errors in the spectral modulus estimate are reasonably small, despite the noise. The errors in the phase estimate appear to be small at low frequencies. They are much larger closer to the folding frequency. This is encouraging for the lightning protection problem, because the most important part of the spectrum lies in the low-frequency region $[20,21,22,23]$. 


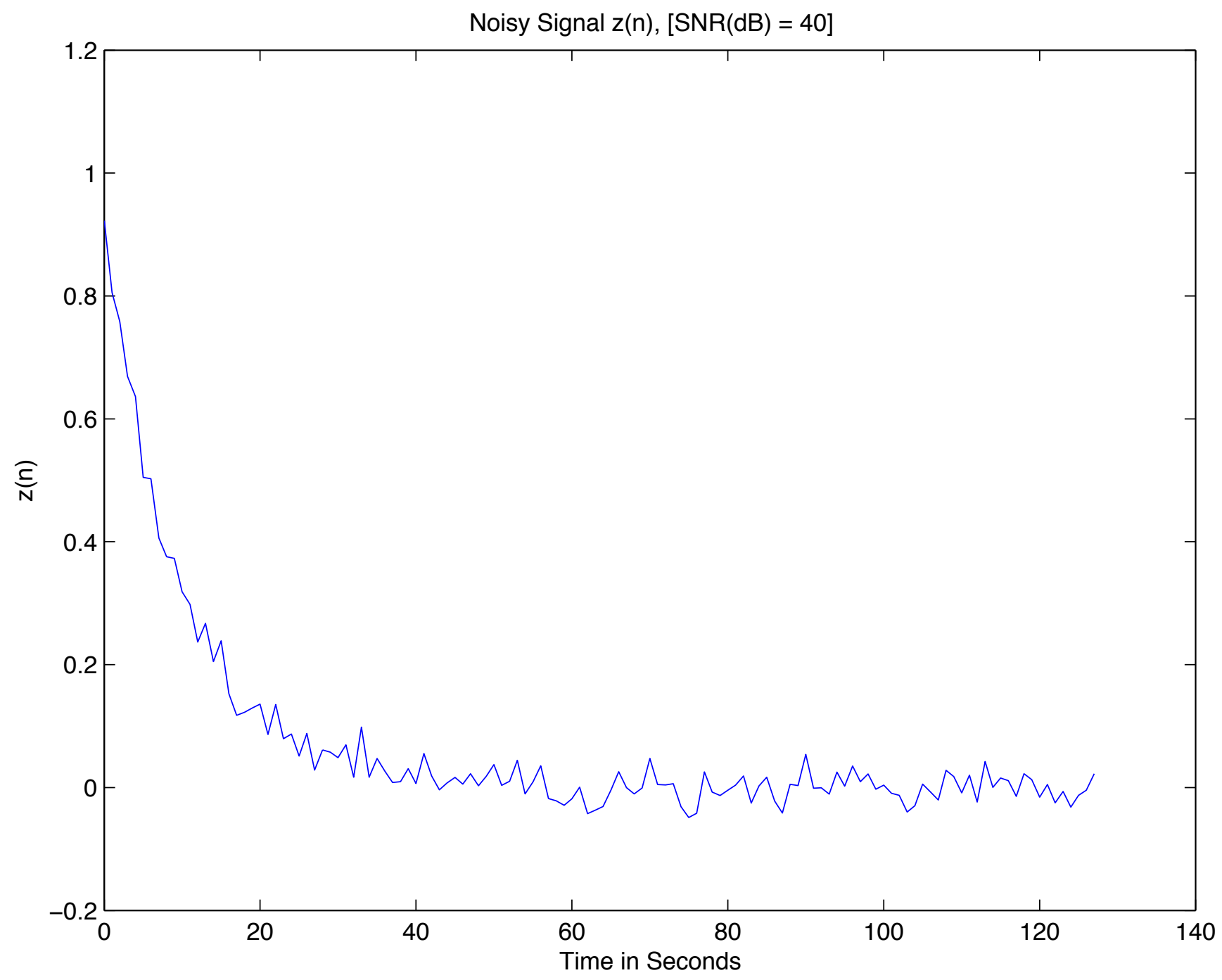

Figure 4.7: The noisy minimum phase signal $\mathrm{z}(\mathrm{n})$ for $\mathrm{SNR}=1 . \mathrm{e} 4(40 \mathrm{~dB})$. 

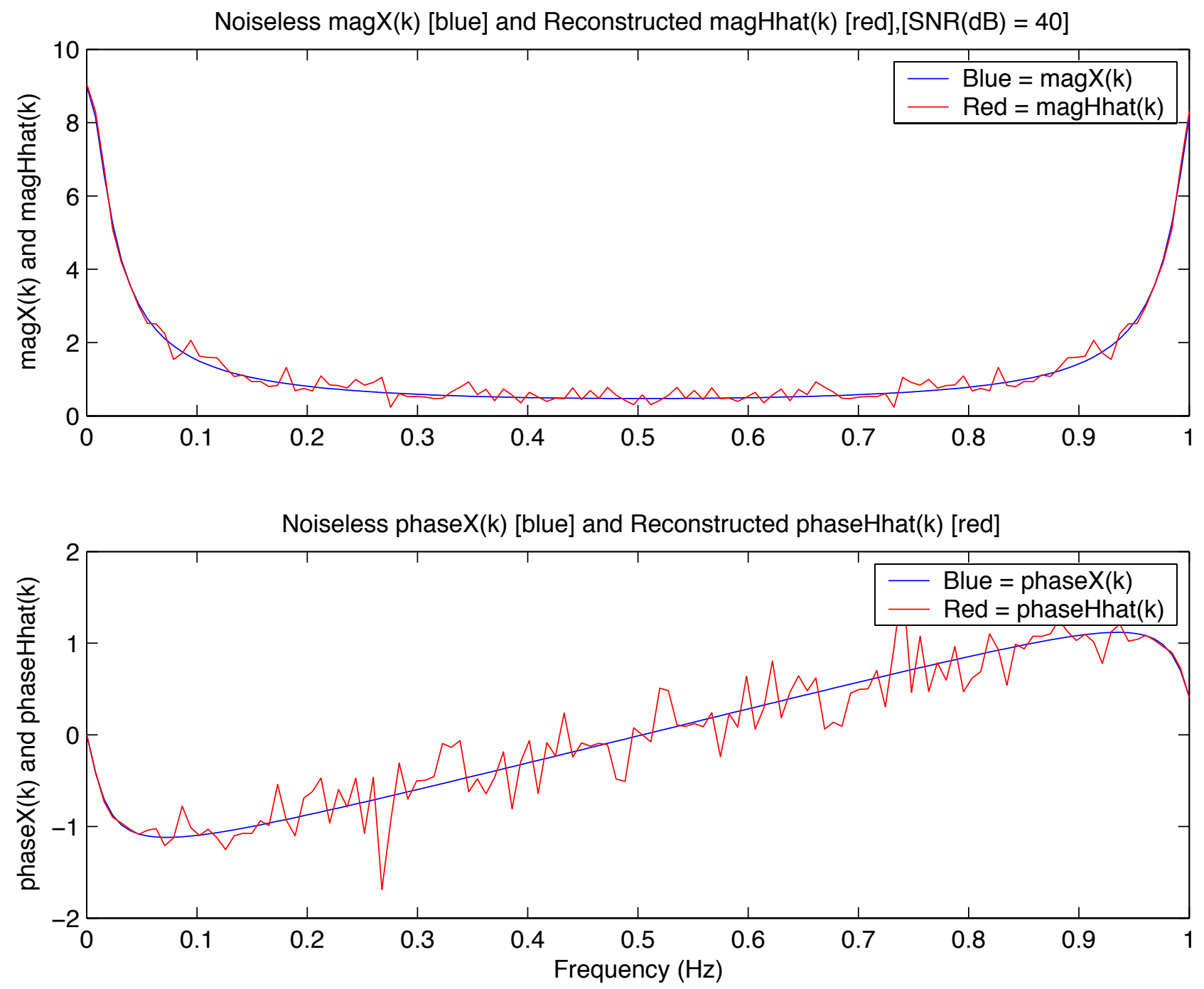

Figure 4.8: The noiseless magnitude and phase of $\mathrm{X}(\mathrm{k})$ and the reconstructed magnitude and phase of $\widehat{H}(k)$ for $\mathrm{SNR}(\mathrm{dB})=40 \mathrm{~dB}$. The errors in the phase estimate appear to be small at low frequencies. They are much larger closer to the folding frequency. 


\section{Chapter 5}

\section{Future Work}

\subsection{Evaluation of the Algorithm Using Measured Lightning Protection Moduli}

Processing real-world signals such as the ones reported in the references $[20,21,22,23]$ with the proposed algorithm will provide definitive information about its efficacy. At this point, a statistical error analysis should be done to quantify the effects of noise, etc. This could include comparing the phase retrieval results with those obtained using the frequency domain division method and the Parks-McClellan FIR filter design algorithm to estimate a minimum phase signal $[16,22]$.

\subsection{Apply Optimal Least-Squares Impulse Response Estimation Algorithms for System Identification}

Currently, the system identification analysis for lightning protection systems is carried out by dividing the moduli of the DFT's of input and output signals. The result is the modulus of a transfer function [20,21, 22, 23]. The This process is suboptimal for many reasons, including the facts that phase is not known (but it is required for system identification), the system identification problem is ill-posed (has no unique solution) and the problem is very sensitive to numerical errors $[17,19]$. We propose that the process can be improved greatly by combining the proposed phase retrieval algorithm in this report with advanced system identification algorithms $[17,19,16]$.

First, reconstruct minimum phase signals for both input and output measurements. Second, carry out the system identification task for Lightning Protection much more effectively using well-known optimal impulse response estimation algorithms $[16,17,19]$. A comparison of the results of such and analysis with the magnitude only results of $[20,21,22,23]$ would provide the ability to make improved evaluations of lightning safety.

\subsection{Phase Retrieval for Partial Moduli}

Sometimes, the lightning protection measurements provide an incomplete spectral modulus. The spectrum analyzer results are sometimes truncated in frequency, so only part of the spectral moduls is available. Systematic methods for coping with this scenario need study. We propose to examine possible approaches, including Bandlimited Spectrum Extapolation algorithms [17]. 


\subsection{R\&D of Generalized Phase Retrieval Algorithms}

In [16], the author proposed R\&D directions. It is recommended that those be pursued. In particular, we are interested in the following generalizations: (1) Algorithms that are not constrained to a minimum phase reconstruction, and (2) Algorithms that are designed to cope with noisy spectral measurements. 


\section{Chapter 6}

\section{Conclusions}

In general, the phase retrieval from modulus problem is very difficult. We exploited the real-and imaginary part sufficiency properties of the Fourier and Hilbert Transforms of causal sequences to develop an algorithm for reconstructing spectral phase given only spectral modulus. The algorithm uses homeomorphic signal processing methods with the complex cepstrum. The development of the algorithm is quite involved, but the final algorithm and its implementation are very simple.

Limitations of the algorithm include the following: (1) It does not account for noise in the given spectral modulus. Fortunately, the lightning protection signals of interest generally have a reasonably high SNR. (2) The DFT length $N$ must be even and larger than the length of the nonzero part of the measured signals. These constraints are simple to meet in practice. (3) Regardless of the properties of the actual signal $h(n)$, the phase retrieval results are constrained to have the minimum phase property. In most problems of practical interest, these assumptions are very reasonable and probably valid. They are reasonable assumptions for Lightning Protection applications.

The efficacy of the theory was demonstrated using simulated signals that meet the assumptions of the algorithm. We saw that for the noiseless case, the reconstructions are extremely accurate. When moderate to heavy simulated white Gaussian noise was added, the algorithm performance remained reasonably robust, especially in the low frequency part of the spectrum, which is the part of most interest for lightning protection.

Proposed future work includes (a) Evaluating the efficacy of the algorithm with real Lightning Protection signals from programmatic applications, (b) Performing a more rigorous analysis of noise effects, (c) Using the algorithm along with advanced system identification algorithms to estimate impulse responses, (d) Developing algorithms to deal with measured partial spectral moduli, and (e) R \& D of phase retrieval algorithms that specifically deal with general (not necessarily minimum phase) signals, and noisy spectral moduli. 


\section{Bibliography}

[1] A. V. Oppenheim and R. W. Schafer,Digital Signal Processing, Prentice-Hall, 1975.

[2] L. R. Rabiner and B. Gold, Theory and Application of Digital Signal Processing, Prentice-Hall, 1975.

[3] A. Whalen, Detection of Signals in Noise Academic Press, 1971.

[4] C. D. McGillem and G. R. Cooper, Continuous and Discrete Signal and System Analysis, Holt, Rinehart and Winston, 1974.

[5] J. V. Candy, Signal Processing, The Modern Approach, McGraw-Hill, 1988.

[6] A. V. Oppenheim, A. S. Willsky and I. T. Young, Signals and Systems, Prentice-Hall, H.J., 1983.

[7] Edited by the Digital Signal Processing Committee of ASSP, Programs for Digital Signal Processing, IEEE Acoustics, Speech and Signal Processing Society, IEEE Press, 1979.

[8] R. Bracewell, The Fourier Transform and its Applications, McGraw-Hill, 1965.

[9] R. E. Crochiere and L. R. Rabiner, Multirate Digital Signal Processing, Prentice-Hall, 1983.

[10] B. P. Bogert, M .J. R. Healy, and J. W. Tukey, The Quefrency Alanysis of Time Series for Echoes: Cepstrum, Pseudo-autocovariance, Cross-Cepstrum, and Saphe Cracking, Proc. Symp. Time Series Analysis, M. Rosenblatt, Ed., New York, John Wiley and Sons, Inc., New York, 1963, pp. 209-243.

[11] A. V. Oppenheim and R. W. Schafer, Homomorphic Analysis of Speech, IEEE Trans. Audio Electroacoustics, Vol. AU16, No. 2, June 1968, pp.221-226.

[12] T. J. Ulrych, Application of Homomorphic Deconvolution to Seismology, Geophysics, Vol. 36, No. 4, August 1971, pp. 650-660.

[13] T. G. Stockham, Jr., Restoration of Old Acoustic Recordings by Means of Digital Signal Processing, Preprint, 41st Convention, Audio Engineering Society, New York, October 1971.

[14] G. A. Clark, Practical Aspects of Sampling and the Discrete Fourier Transform, LLNL Continuing Education Series, November 1983, with ongoing presentations over the past two decades.

[15] G. A. Clark, Elements of Digital Signal and Image Processing - Practical and Theoretical Preparation for MATLAB Training, Electronics Engineering Dept., Defense Sciences Engineering Division, Lawrence Livermore National Laboratory, UCRL-PRES-154699, September 2003.

[16] G. A. Clark, Signal Processing Analysis and Recommendations for Lightning Protection Projects, Lawrence Livermore National Laboratory Report UCRL-PRES-xxxxx (Not yet through Review and Release), January 14, 2004.

[17] G. A. Clark, D.M. Tilly, and W. D. Cook, Ultrasonic Signal/Image Restoration for Quantitative NDE, NDT International, Vol. 19, No. 3, June 1986. 
[18] G. A. Clark and E. J. Bogdan, Summary of Rules for Sampling, Decimation and Interpolation of Experimental Data, Lawrence Livermore National Laboratory Report UCRL-19411, April 1982.

[19] J. V. Candy, G. A. Clark, and D. M. Goodman, Transient Electromagnetic Signal Processing: An Overview of Techniques, Chapter in the book, Time Domain Measurements in Electromagnetics, edited by Edmund K. Miller, Van Nostrand Reinhold Co., New York, 1986, pp. 416-455.

[20] M. M. Ong and C. G. Brown Jr., Completion of Action Items from Lightning Protection Review, Lawrence Livermore National Laboratory document AE and GOG 020304, February 3, 2004.

[21] T. J. Clancy, M. M. Ong, Lightning Protection System, LLNL Document, September 11, 2003.

[22] T. J. Clancy, C. G. Brown, M. M. Ong, E. Baluyot, Lightning Assessment and Certification of H.E. Storage and Processing Facilities at Site 300, Formal Design Review for Quality Assurance, Lawrence Livermore National Laboratory Document, December 12, 2003.

[23] C. G. Brown, Phase Retrieval in Lightning Safety Assessment Measurements, Lawrence Livermore National Laboratory Document, December 4, 2003.

[24] A. Karwowski and A. Zeddam, Transient Currents on Lightning Protection Systems Due to the Indirect Lightning Effect, Science, Measurement and Technology, IEE Proceedings, Vol. 142 , Issue 3 , May 1995 Pages 213 - 222.

[25] G. Bachman, L. Narici, Functional Analysis, Academic Press, 1966.

[26] B. Noble, Applied Linear Algebra, Prentice-Hall, 1969.

[27] R. V. Churchill Complex Variables and Applications, McGraw-Hill, 1960.

[28] MATLAB Reference Manual, The Mathworks, Natick Massachussetts, 1993. 


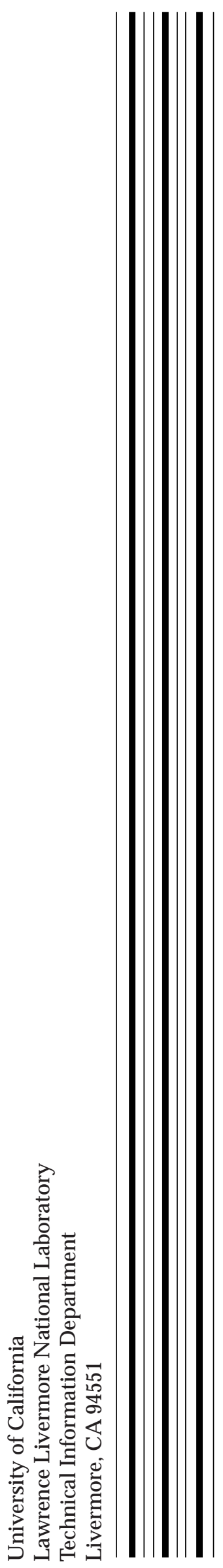

